\title{
Digitalisierung: Perspektiven für Arbeitsmodelle der Zukunft in Wirtschaft und Verwaltung
}

\author{
Jochen Schellinger und Gia Le Huynh
}

\begin{abstract}
Zusammenfassung
Die Digitalisierung ist derzeit einer der wichtigsten Wandlungstreiber in Wirtschaft und Gesellschaft. Die aus den neuen technischen Möglichkeiten resultierenden Veränderungen wirken sich auch auf das Verhalten von Kunden und Mitarbeitenden aus. In Bezug auf die Zusammenarbeit in Unternehmen und Organisationen sind bestehende Arbeitsmodelle für administrative Arbeit anzupassen und neue Formen digitaler Kollaboration einzuführen. Hiermit verbunden sind Änderungen der Arbeitszeitmodelle, der Organisation der Mitarbeitenden, der Art der Zusammenarbeit und Führung sowie der Gestaltung von Büroräumlichkeiten. In einem vergleichenden Case-Study-Ansatz von zwei Schweizer Großunternehmen und einer großen Verwaltungseinheit des Bundes werden die existierenden Arbeitsmodelle für Büroarbeit analysiert und auf Entwicklungsperspektiven hin untersucht. Ein derzeit vorhandener Mix aus traditionellen und agilen Arbeitsmodellen wird sich in Zukunft im digitalen Kontext voraussichtlich unternehmensspezifisch stärker in Richtung einer höheren Agilität weiterentwickeln.
\end{abstract}

J. Schellinger $(\bowtie) \cdot$ G. Le Huynh

Berner Fachhochschule, Departement Wirtschaft, Bern, Schweiz

E-Mail: jochen.schellinger@bfh.ch

G. Le Huynh

E-Mail: gia-le.huynh@gmx.net 


\subsection{Einleitung}

Die Arbeitswelt befindet sich im Wandel. Globalisierung, die Alterung der Gesellschaft, die Pluralisierung von Lebensmodellen, aber auch die Digitalisierung treiben diesen Wandel weiter voran. Insbesondere Letztere verändert die Struktur der Arbeitswelt grundlegend (Neufeind 2016, S. 58). Damit Unternehmen die mit der Digitalisierung verbundenen Herausforderungen bewältigen können, braucht es stimmigere und agilere Arbeitsmodelle und Strukturen (Schweizer Verband der Telekommunikation 2015, S. 20 f.). In der Literatur finden sich bislang nur wenig konkrete Aussagen über die Auswirkungen der Digitalisierung für die Implementierung praxistauglicher Arbeitsmodelle für administrative Arbeit. Ein deskriptives Herangehen in Bezug auf aktuelle Umsetzungsmodelle in sich entwickelnden größeren Organisationen soll dabei helfen, denkbare Zukunftsszenarien digitaler Arbeitswelten zu entwickeln. Im Mittelpunkt des Interesses steht die Frage nach dem Aussehen zukünftig erfolgversprechender Arbeitsmodelle für Büroarbeit. Hiermit verbunden sind Fragestellungen hinsichtlich der gegenwärtigen Ausprägung erfolgswirksamer Arbeitsmodelle, der Digitalisierungschancen und -risiken, der konkreten Veränderungsbedarfe, relevanter Rahmensetzungen sowie der Implementierung neuer, beziehungsweise veränderter Arbeitsmodelle.

\subsection{Gegenstand und allgemeine Auswirkungen der Digitalisierung auf Großunternehmen}

Eine Analyse der Auswirkungen von Digitalisierung auf Arbeitsmodelle setzt zunächst eine Klärung von Gegenstand, Antriebskräften und möglichen Auswirkungen der Digitalisierung voraus. Ferner stellt sich die Frage, ob und wie der Digitalisierungsgrad von Unternehmen gemessen werden kann und welche Potenziale die Digitalisierung für die hier im Vordergrund stehenden Großunternehmen und -organisationen mit sich bringt.

\subsubsection{Begriff und Eigenschaften der Digitalisierung}

Der Begriff „Digitalisierung“ wird in der Literatur sehr vielfältig verwendet und interpretiert. Er wird häufig synonym zu den Begriffen „digitale Transformation“ und „digitales Zeitalter" verwendet. Der Begriff steht insbesondere für die digitale Vernetzung aller Bereiche in Wirtschaft und Gesellschaft sowie für die durch die Digitalisierung ausgelösten Veränderungsprozesse (Schallmo und Rusnjak 2017). Grundlage der Digitalisierung sind digitale Technologien für die Datengewinnung, -erfassung, -speicherung und die technische Datenverarbeitung (Blaeser-Benfer und Pollety 2017, S. 14). In Verbindung mit menschlicher Arbeit werden in der Unternehmenspraxis vielfach mit dem Begriff digitale Arbeitsprozesse und -inhalte, eine Beschleunigung der Arbeit und mobil-flexible und papierlose Arbeitsformen verbunden und mit digitalen Phänomenen 
wie Social Media, Big Data, künstlicher Intelligenz oder Industrie 4.0 in Verbindung gebracht (Genner et al. 2017, S. 4 f.; Ittermann und Niehaus 2015, S. 34 f.). Insbesondere der letzte Begriff steht auch als Synonym für eine vierte industrielle Revolution hin zur Wissens- und Informationsgesellschaft, einer neuen Ära der digitalen Automatisierung durch die Mustererkennung in riesigen Datenmengen, cloudbasierte Verfahren, Internet of Things, Smart-Industry-Konzepte, Robotik, Maschinenlernen, Sensorik u. a. Digitalisierung kann aber auch im eher evolutionären Sinne lediglich als Fortführung einer zunehmenden Computerisierung verstanden werden (Raveling 2017b; Schwab 2016, S. $17 \mathrm{f}$.).

\subsubsection{Treiber und Auswirkungen der Digitalisierung}

Disler (2018) identifiziert insgesamt sechs primäre Treiber der Digitalisierung, die nachfolgend eingehender betrachtet werden:

- Globalisierung.

- New Economy.

- Internet of Things.

- Big Data.

- Kunden.

- Transaktionskosten.

Die „Globalisierung“ steht für die wachsende weltweite Verflechtung von Wirtschaft, Politik, Kultur sowie Umwelt und den weltweiten Austausch von Produkten, Ressourcen, Technologien und Kapital. Die Globalisierung fördert die Agilität in allen Bereichen der Volkswirtschaft. Der Begriff „New Economy“ versinnbildlicht den Fokuswechsel weg von der Warenproduktion hin zu webbasierten Dienstleistungen. Das „Internet of Things (IoT)“" verbindet die virtuelle mit der physischen Welt und ermöglicht ein Netzwerk aus Geräten, die miteinander weltweit kommunizieren und interagieren können (Machine-to-Machine-Funktion). Mit „Big Data“ verbindet man die Wertgenerierung aus der Verarbeitung und Analyse riesiger Datenmengen, z. B. um zielgruppenorientierte Kundenbedarfe zu identifizieren oder Absatzmengen vorherzusagen. Zudem hat sich das Konsum- und Kaufverhalten in den letzten Jahren massiv verändert: Die „Kunden“ treiben die Digitalisierung voran, indem sie neue Anforderungen an Transparenz, Kauferlebnisse und die Geschwindigkeit von Lösungen und Services stellen. „Transaktionskosten“ sind Kosten, die durch die Nutzung des Marktes im Zusammenhang mit der Transaktion von Verfügungsrechten entstehen. Vereinfachungen von Abläufen und Konzentration auf Kundenbedürfnisse dienen u. a. der Reduktion der Transaktionskosten (Disler 2018). Vor allem diese sechs Digitalisierungstreiber haben dazu geführt, dass der Digitalisierungsprozess in der Unternehmenswelt, aber auch in administrativen serviceorientierten Organisationen, mit hoher Dynamik und wachsendem Wirkungskreis vorangeht. 
Die Digitalisierung und die hinter ihr stehenden Treiber haben große Auswirkungen auf die Unternehmen. Veränderten Kundenerwartungen kann durch datenbasierte, transparenzschaffende Analyseverfahren besser erkannt und begegnet werden. Dies geht gleichzeitig mit einer Machtverschiebung in Richtung Verbraucher einher (Schwab 2016, S. 82 f.). Erweiterungen von Produkten um digitale Fähigkeiten führen ferner zu Kundennutzensteigerungen und ermöglichen neue Geschäftsmodelle (Schwab 2016, S. 85 f.). Angesichts der Schnelligkeit, Innovation und Disruption, die die neuen Technologien mit sich bringen, braucht es neue Formen der kooperativen Zusammenarbeit mit Partnern. Es ist von Bedeutung, Kundenerfahrungen, datenbasierte Dienstleistungen sowie Leistungsbeurteilungen gegenseitig auszutauschen (Schwab 2016, S. 87 f.). Durch neue Technologien, geänderte Produktionsverfahren und veränderte Kundenerwartungen müssen Unternehmen ihre Geschäftsmodelle überdenken. In Verbindung mit relevanter werdenden Plattformstrategien erfolgen zunehmend Schwerpunktverlagerungen weg vom Produktverkauf hin zum Erbringen von Dienstleistungen (Schwab 2016, S. 89).

Die Digitalisierung hat auch fundamentale Auswirkungen auf die Arbeitswelt und somit auf die Arbeitnehmer und wirkt über die Unternehmenswelt hinaus zudem in das Privatleben der Beschäftigten hinein. Digitale Technologien und Kommunikationskanäle sind für die meisten Menschen mittlerweile auch in der Freizeit über Smartphones, Tablets oder Social Networking zum festen Lebensbestandteil geworden (Glaser 2016). Wie wir einkaufen, wie wir Musik hören und wie wir unser Leben gestalten, das alles wird durch die Digitalisierung beeinflusst (Süddeutsche Zeitung, o. J.). Angesichts der neuen Kommunikationsmöglichkeiten und der jederzeitigen Erreichbarkeit der Beschäftigten vermischen sich Arbeitswelt und Freizeit in zunehmendem Maße und die Grenzziehung zwischen diesen Sphären wird aufgeweicht.

\subsubsection{Ansätze zur Messung der Digitalisierung}

Zur Bewertung des Digitalisierungsgrads von Unternehmen und Organisationen können Reifegradmodelle herangezogen werden. Es handelt sich dabei um Referenzmodelle, die sich mithilfe von definierten Entwicklungsstufen (Reifegrade) mit dem aktuellen Entwicklungszustand, Änderungen in Organisationen sowie mit den eingesetzten Technologien befassen (Leyh et al. 2016, S. 3). Die Reifegradmessung hilft, ein gemeinsames Verständnis für die Digitalisierung herzustellen. Sie hilft bei der Standortbestimmung und Aufdeckung von Wahrnehmungsdifferenzen und ermöglicht Vergleiche mit anderen Unternehmen (Berghaus et al. 2017, S. 7).

Es gibt mittlerweile eine Reihe von Reifegradmodellen zur Messung der digitalen Reife von Unternehmen, die sich in Art und Umfang der herangezogenen Transformationsfaktoren, respektive Digitalisierungsmerkmale unterscheiden (Berlin 2016, S. 6). Die beiden wohl bekanntesten wissenschaftlichen Verfahren sind der „Four-Types-of-Digital-Maturity“-Ansatz des Centers for Digital Business am Massachusetts Institute of Technology (MIT) und das „Digital Transformation Assessment“ von Forrester Research 
(Digital Transformer Institute 2017; Ruoss 2015). Das „Digital Maturity Model“ der Universität St. Gallen (Berlin 2016, S. 6) scheint im Besonderen für die spezifischen Rahmenbedingungen in der Schweiz geeignet zu sein. Das Institut für Wirtschaftsinformatik der Universität St. Gallen und die Strategieberatung Crosswalk untersuchen seit 2014 die digitale Reife von Unternehmen in der Schweiz, Deutschland und Österreich. Zwischenzeitlich nehmen 452 Unternehmen an der Studie teil (Institut für Wirtschaftsinformatik 2017; Berghaus et al. 2017, S. 6, 11). Das „Digital Maturity Model“ besteht aus den neun Dimensionen Customer Experience, Produktinnovation, Strategie, Organisation, Prozessdigitalisierung, Zusammenarbeit, Informationstechnologie, Kultur \& Expertise sowie Transformationsmanagement, die mittels 64 Indikatoren in Online-Fragebögen erhoben werden (Berghaus et al. 2017, S. 8). Der Reifegrad eines Unternehmens wird in drei Schritten mittels Indikatoreneinstufung auf einer fünfstufigen Likert-Skala, einer Schwierigkeitsgradbewertung pro Indikator und einer Clusterbildung ermittelt (Berghaus et al. 2017, S. 8 f.).

\subsubsection{Potenziale der Digitalisierung für Großunternehmen}

Großunternehmen haben gegenüber Klein- und mittleren Unternehmen (KMU) nicht nur mehr Beschäftigte, sondern verfügen auch über mehr Ressourcen und Handlungsmöglichkeiten. Sie weisen zudem in der Regel einen höheren digitalen Reifegrad als kleinere Unternehmen auf und eignen sich somit besser für die Analyse der digitalisierungsbasierten Veränderung von Arbeitsmodellen.

Es gibt in der Schweiz rund 1570 Großunternehmen, welche 1,4 Mio. Personen beschäftigen, was einem Anteil von rund $30 \%$ der Beschäftigten entspricht. Als Großunternehmen gilt in der Schweiz ein Unternehmen, welches mindestens 250 Personen beschäftigt (Schweizerische Eidgenossenschaft 2018a; Moneyhouse 2017). Losgelöst von der Unternehmensgröße wenden viele Unternehmen bereits digitale Technologien an, um die Effizienz sowie den Mehrwert ihrer Produkte und Dienstleistungen zu verbessern. Schweizer Unternehmen sehen in Verbindung mit der Digitalisierung insbesondere folgende Potenziale zur Verbesserung ihrer Unternehmensperformance (Deflorin et al. 2015, S. 58 ff.):

- Effizienzsteigerung und Automatisierung von Produktion und Prozessen.

- Überwachung von Prozessen.

- Verbesserung von Dienstleistungsmehrwert und Dienstleistungsangebot.

- Verbesserung von Produktmehrwert sowie Einführungszeit von Produkten.

- Einbettung von digitalen Schnittstellen in die Produkte für die Erhöhung von Marktund Kundenwissen.

- Gemeinsame digitale Plattformen für unterschiedliche Funktionsbereiche, wie beispielsweise Forschung und Entwicklung, Produktion sowie Marketing.

- Erhöhung der Integration von Funktionen und Prozessen.

- IT-Schnittstellen zu externen Partnern in der Wertschöpfungskette, auch international. 
Aus den Potenzialen können kompetitive Chancen, wie Kostensenkungen, vermehrte Kundenbindung sowie individualisierte neue Produkte und Dienstleistungen entstehen (Deflorin et al. 2015, S. 61; Deloitte 2016, S. 6). Hierfür müssen Unternehmen ihre Geschäftsstrategie und -ziele anpassen, digitale Initiativen starten und adäquate Strukturen und Arbeitsmodelle sowie eine Innovationskultur einführen (Deflorin et al. 2015, S. 61; Deloitte 2016, S. 17). Eine explizit auf Großunternehmen mit mehr als 500 Mitarbeitenden beschränkte Studie in Deutschland kommt zu ähnlichen Ergebnissen. Das Potenzial der Digitalisierung wird von diesen Großunternehmen aber als noch größer eingeschätzt als von KMU (Rotter 2017).

Die jüngsten Ergebnisse des Digital Maturity Report können Hinweise darauf geben, welche Herangehensweisen für Großunternehmen in der Schweiz bei der Digitalisierung relevant sein können, auch wenn die Untersuchung nicht nur auf diese Unternehmenskategorie ausgerichtet ist (Berghaus et al. 2017, S. 17). Es konnten fünf typische Vorgehensweisen zur Digitalisierungsumsetzung identifiziert werden (Berghaus et al. 2017, S. 20 f.):

- Top-down: Die Digitalisierung wird von der Führungsebene getrieben. Das Hauptaugenmerk wird dabei auf die Erstellung und Umsetzung einer digitalen Transformationsstrategie gesetzt.

- Bottom-up: Die Mitarbeitenden ergreifen Initiativen und konzentrieren sich auf die Konsolidierung von bestehenden Initiativen.

- IT-Fokus: Die Anforderungen an die IT sind Treiber der Digitalisierung. Im Mittelpunkt stehen die Erneuerung und Bereitstellung geeigneter IT-Infrastruktur und -Systeme.

- Kanal-Fokus: Das Unternehmen wird von den digitalen Kundenerwartungen getrieben. Hierbei wird auf die Erneuerung und Verbesserung der digitalen Kanäle fokussiert.

- Innovations-Fokus: Digitalisierung wird durch Experimentierfreude oder Gefährdung des aktuellen Geschäftsmodells vorangetrieben. Das Ausprobieren neuer Technologien und die Erarbeitung möglicher neuer Geschäftsmodelle sind dabei zentral.

Von den in der Studie befragten 662 Unternehmen wählt das Gros von $23 \%$ einen Top-Down-Ansatz (Berghaus et al. 2017, S. 20 f.). Ferner sind Unternehmen mit niedrigem Digitalisierungsreifegrad häufig IT-fokussiert oder wenden den Bottom-Up-Ansatz an. Unternehmen mit hohem Reifegrad präferieren eine Top-downoder innovationsfokussierte Herangehensweise vor (Berghaus et al. 2017, S. 20 f.). Das Gros der Unternehmen weist auf der fünfstufigen Reifegradskala einen Wert von 3 auf, der durchschnittliche Reifegrad der 452 einbezogenen Unternehmen liegt bei 2,8. Großunternehmen mit mehr als 10.000 Angestellten verfügen über die höchsten Digitalisierungsreifegrade. Großunternehmen mit einer Beschäftigtenzahl von 251 bis 500 Mitarbeitenden liegen aber nahezu gleichauf. Insgesamt liegen die Reifegrade der Großunternehmen leicht oder spürbar über dem durchschnittlichen Reifegradswert 
(Berghaus et al. 2017, S. 18). Branchenbezogen liegen die IT, Telekommunikation \& Beratung, Versicherungen sowie die Banken mit einem durchschnittlichen Reifegrad ab 3 im vorderen Bereich der Digitalisierungsimplementierer (Berghaus et al. 2017, S. 19).

\subsection{Arbeitsmodelle für administrative Arbeit in der Schweiz}

In den folgenden Abschnitten werden Arbeitsmodelle im Schweizer Kontext inhaltlich anhand von fünf Gestaltungsdimensionen charakterisiert und die wichtigsten gesetzlichen Rahmenbedingungen für die Ausgestaltung von Arbeitsmodellen für administrative Arbeit aufgezeigt.

\subsubsection{Begriff und Gegenstand von Arbeitsmodellen}

Auch der Begriff „Arbeitsmodell“ ist in der Literatur vielfältig belegt. Oft wird er mit Arbeitszeitmodellen gleichgesetzt, was jedoch der heutigen veränderten Arbeitswelt mit einer Verschmelzung von Arbeitszeit-, Arbeitsform-, Arbeitsführungs-, Arbeitsstrukturund Arbeitsraumdimensionen nicht mehr gerecht wird. Arbeitsmodelle im Verständnis des vorliegenden Beitrags umfassen alle diese dimensionalen Ausprägungen von (Zusammen-)Arbeit. In der nachfolgenden Kennzeichnung dieser Dimensionen wird der Fokus auf in diesem Beitrag im Vordergrund stehende administrative Tätigkeiten gelegt.

\subsubsection{Arbeitszeitdimension von Arbeitsmodellen}

Die Arbeitszeitdimension kann anhand der Hauptausprägungen von Arbeitszeitmodellen charakterisiert werden. Im Digitalisierungskontext kommt vor allem auf Flexibilität und Eigenverantwortung setzenden Zeitmodellen eine größere Bedeutung zu. Bei der stark verbreiteten Vertrauensarbeitszeit verzichten Arbeitgeber auf Zeitkontrollen. Die Mitarbeitenden handeln eigenverantwortlich und orientieren sich am Resultat ihrer Arbeit. Bei der Umsetzung sind Restriktionen der Arbeitsgesetzgebung zu berücksichtigen, was dazu führt, dass Vertrauensarbeitszeitmodelle vor allem bei Führungskräften zum Einsatz kommen (Lienhart 2015). In flexiblen Arbeitszeitmodellen können die Beschäftigten in einem bestimmten Rahmen selbst auf ihre Arbeitszeit Einfluss nehmen. Das bekannteste, in der Praxis bewährte und beliebte Modell ist das Gleitzeitmodell. Es lässt sich gut mit den arbeitsrechtlichen Vorschriften vereinbaren. Ein Gleitzeitreglement regelt, dass sowohl durch Minussaldo als auch Plussaldo Abweichungen von der durchschnittlichen Arbeitszeit entstehen können und wie diese ausgeglichen werden. Gleitzeitregelungen sorgen in erster Linie dafür, dass für Arbeitnehmer und für Arbeitgeber mehr Flexibilität entsteht. Amorphe Arbeitszeitmodelle sind dadurch gekennzeichnet, dass für Mitarbeitende lediglich festgehalten wird, wie viel in einem bestimmten Zeitraum gearbeitet 
werden muss, aber nicht, wann und wie lange gearbeitet wird (Wochen-, Monats-, Jahres- und Lebensarbeitszeitmodelle). Zu viel geleistete Arbeitszeit wird auf einem Arbeitszeitkonto festgehalten und kann für Sabbaticals, Familienpausen oder eine vorzeitige Pensionierung angespart werden. Die Mehrarbeit der Mitarbeitenden stellt eine geldwerte Leistung dar und muss in der Bilanz des Unternehmens als Schuld ausgewiesen werden. Viele Unternehmen wählen diese Modellvariante wegen dieser Offenlegungspflicht nicht. Jahresarbeitszeit kommt vor allem bei Unternehmen mit starken Schwankungen im Arbeitsanfall zum Tragen. Lebensarbeitszeitmodelle sind Weiterführungen des Jahresarbeitszeitmodells mit weitergehenderen Kompensationsoptionen. Sie kommen in der Schweiz vor allem in der öffentlichen Verwaltung zur Anwendung (Arbeitgeberverband Basel 2014).

\subsubsection{Arbeitsformdimension von Arbeitsmodellen}

Unter Arbeitsformen werden hier das Jobsharing, die Teilzeitarbeit sowie die Arbeit auf Abruf subsumiert. Den angeführten Arbeitsformen kommt hinsichtlich der im Digitalisierungskontext besonders wichtigen Flexibilisierung und Mobilisierung der Arbeit eine größere Relevanz zu.

Jobsharing ist dadurch gekennzeichnet, dass eine Arbeitsstelle auf mehrere Arbeitnehmer aufgeteilt wird und die zugehörigen Aufgabenstellungen gemeinsam, aber zeitversetzt als Gruppe bewältigt werden. Die Jobsharer arbeiten teilzeit und können die Arbeitszeit im definierten Rahmen selber einteilen. Hauptmerkmal der Teilzeitarbeit ist eine gegenüber der betriebsüblichen Arbeitszeit reduzierte Arbeitszeit. Oft arbeiten Teilzeitbeschäftigte für mehrere Arbeitgeber unter der Voraussetzung, dass nicht gegen die Sorgfalts- und Treuepflicht verstoßen wird. Bei der Arbeit auf Abruf veranlasst der Arbeitgeber den Einsatz der Beschäftigten bedarfsabhängig. Hierbei sind Abrufarbeiten mit und ohne Befolgungspflicht zu unterscheiden. Beim Abruf mit Befolgungspflicht sind Mitarbeitende verpflichtet dem Abruf zu folgen, und die Beschäftigten müssen auch außerhalb der betrieblichen Arbeitszeit für einen Arbeitseinsatz zur Verfügung stehen (entschädigungspflichtiger Bereitschaftsdienst). Eine Abrufarbeit mit Befolgungspflicht ist in der Regel auch eine Teilzeitarbeit. Bei der Abrufarbeit ohne Befolgungspflicht können Beschäftigte frei entscheiden, ob sie dem Abruf Folge leisten oder nicht. Prinzipiell entsteht dabei durch jeden neuen Arbeitseinsatz ein neuer Vertrag (Bürgi und Nägeli Rechtsanwälte o. J.).

\subsubsection{Arbeitsführungsdimension von Arbeitsmodellen}

Je nach Arbeitssituation und Personenkreis kommen in den Unternehmen heutzutage verschiedene Personalführungsansätze zur Anwendung. In der Literatur gibt es eine Fülle von Führungsmodellen, die sich in der praktischen Anwendung im Laufe der vergangenen 
Jahrzehnte zwar hinsichtlich Schwerpunktsetzung und Variabilität verändert haben, in ihrer Grundform aber eine hohe Kontinuität vorweisen. Eine bekannte klassische Grundkategorisierung von Führungsansätzen ist das „Kontinuummodell“ nach Tannenbaum und Schmidt (Grannemann 2015). Es ordnet die in Anlehnung an Lewin abgegrenzten Führungsstile entlang eines Kontinuums, das durch zunehmende Entscheidungsspielräume der Gruppe und abnehmende Entscheidungsspielräume der Vorgesetzten gekennzeichnet ist. Die Bandbreite der Personalführungsperspektive reicht hier von autoritären, über konsultative, partizipative bis hin zu kooperativ-demokratischen Entscheidungsfindungsmodellen (Grannemann 2015). Im Zuge der Digitalisierung gewinnen Gruppenansätze noch mehr an Bedeutung, sodass im Rahmen eines situativen Führungsansatzes vermehrt kooperative, oder, noch weitergehend, auf Selbstorganisation setzende, vertrauensbasierte laterale Führungsmodelle an Bedeutung gewinnen.

\subsubsection{Arbeitsstrukturdimension von Arbeitsmodellen}

Arbeitsmodelle sind immer auch untrennbar mit der Frage nach strukturellen Festlegungen zur Zusammenarbeit verbunden. Die Grundkategorisierung von in der Unternehmenspraxis vorhandenen Organisationsformen nach Sattelberger et al. (2015) gibt einen Überblick über vier Basisformen zur Organisation der Arbeit, die sich durch ihr Organisationsdesign und die Rollen der Mitarbeitenden unterscheiden. Das Organisationsdesign kann gemäß dem Typologieschema gesteuert oder selbstorganisierend ausgerichtet werden und die Rollen der Mitarbeitenden werden entweder als umsetzungsorientiert oder als gestaltungsorientiert beschrieben (Sattelberger et al. 2015, S. 270 ff.). Je nach Kombination dieser zweidimensionalen Ausprägungsformen resultieren vier Organisationsformen, die in Verbindung mit der Digitalisierung der Arbeitswelt mehr oder weniger gut geeignet sein können.

Gesteuerte und umsetzungsorientierte Weisungs- und Kontrollorganisationen haben eine lange Tradition und sind vor allem dann geeignet, wenn man durch Routineprozesse Effizienz generieren möchte. In der Top-Down-Struktur stehen Mitarbeitende im Vordergrund, die sich durch effiziente Ausführungen vorgegebener Prozesse auszeichnen. Kreativität und Innovation werden durch Hierarchie und Standardisierung allerdings nicht gefördert (Sattelberger et al. 2015, S. 271 f.). Selbstorganisierende und umsetzungsorientierte überlastete Organisationen sind dadurch gekennzeichnet, dass veränderte Marktbedürfnislagen dazu geführt haben, dass anstelle von nur ausführenden Mitarbeitenden von diesen zunehmend mehr Eigenverantwortung, innovatives Verhalten und Selbstorganisation der Arbeit erwartet wird. Dies funktioniert jedoch nur, wenn die Mitarbeitenden hierfür befähigt werden, was in der Praxis oft nicht der Fall ist und dann zu Überbelastung der Beschäftigten führt (Sattelberger et al. 2015, S. 272). Ähnlich problematisch können sich gesteuerte und gestaltungsorientierte Schattenorganisationen entwickeln, die durch Mitarbeitende gekennzeichnet sind, die sich aktiv als Mitentscheider am Unternehmenserfolg beteiligen möchten, jedoch von den vorhandenen 
autoritären, starren Strukturen ausgebremst werden. Mitarbeitende verfolgen in diesem Umfeld zunehmend ihren eigenen Weg und entwickeln oftmals eine hohe Eigendynamik, die nur bedingt mit den strategischen Rahmensetzungen kompatibel sein muss. Oft verlassen gute und an sich motivierte Mitarbeitende solche Organisationen (Sattelberger et al. 2015, S. 274-275). Selbstorganisierende und gestaltungsorientierte agile Netzwerke verfügen über ein flexibles vertrauensbasiertes Organisationsdesign und eigenverantwortliche Mitarbeitende mit unternehmerischen Gestalterqualitäten. Mitarbeitende werden möglichst umfassend bei wichtigen Entscheidungen einbezogen. Sie arbeiten eigenständig, denken unternehmerisch und erschließen und überführen komplexe Sachverhalte in Konzepte (Sattelberger et al. 2015, S. 276 f.). Diese auf Innovation ausgerichtete Organisationsform scheint in Verbindung mit digitalisierungsbedingten Anpassungen von Arbeitsmodellen von besonderer Bedeutung zu sein. Agilität und Digitalisierung sind Begriffe, die in hohem Maße miteinander kompatibel sind.

\subsubsection{Arbeitsraumdimension von Arbeitsmodellen}

Neben der Zeitgestaltung, Arbeitsarten, Führung und der Organisation trägt auch der Arbeitsort im Sinne einer physischen Festlegung dessen, wo und wie Mitarbeitende zusammenarbeiten, zur Produktivität und Zufriedenheit der Angestellten bei. Traditionelle Büroraumlösungen sind dadurch gekennzeichnet, dass sie für Mitarbeitende konzipiert sind, die ihre Arbeiten fix an einem Standort und zu festen Zeiten erledigen. Die Räume sind in der Regel hierarchisch strukturiert. Es gibt Einzel- und Teambüros und jeder Mitarbeitende hat seinen eigenen Arbeitsplatz. Etwa zehn Prozent der Schweizer Unternehmen nutzen noch diese klassische Form der Bürogestaltung. Flexible Büros sind durch flache hierarchische Strukturierungen und einen geringen Anteil von Einzelbüros gekennzeichnet. Homeoffice ist zwar möglich, wird aber nicht wirklich gefördert. Etwa fünf Prozent der Schweizer Unternehmen verwenden heute solche flexibleren Raumlösungen. Mobile Büros, die bei etwa $16 \%$ der Schweizer Unternehmen zum Einsatz kommen, sind auf mobil-flexibles Arbeiten mit geeigneter Infrastruktur ausgerichtet. Hierarchien spielen, wie bei den flexiblen Raumlösungen, eher keine Rolle bzw. sind flach und es werden Open-Space-Lösungen als moderne Form des Großraumbüros implementiert. Zwei Drittel der Schweizer Unternehmen befinden sich entweder im Wechsel in Richtung flexible oder mobile Lösungen oder sind nicht eindeutig einer Kategorie zuordenbar (Witzig the office company 2014).

\subsubsection{Arbeitsrechtliche Rahmenbedingungen}

Bei der Ausgestaltung der Arbeitsmodelle entlang der angeführten Gestaltungsdimensionen im Hinblick auf Aspekte der digitalen Transformation sind die arbeitsrechtlichen Regelungen als Rahmensetzung zu berücksichtigen. Diese sind für die Schweiz 
im Obligationenrecht (OR) und im Arbeitsgesetz (ArG) festgelegt (Schweizerische Eidgenossenschaft o. J.a.). Hinsichtlich Flexibilisierung und Mobilisierung in zukünftigen digitalen Arbeitswelten sind insbesondere die gesetzlichen Regelungen für Überstunden, Nacht- und Sonntagsarbeit, zur Arbeitszeiterfassung und zur Mehrbeschäftigung relevant.

Sofern Arbeitsstunden, die vertraglich vereinbarte Arbeitszeit übersteigen, spricht der Gesetzgeber von Überstunden, die nur in Ausnahmefällen zu leisten sind. Beschäftigte müssen körperlich und psychisch Überstunden leisten können, die Anforderungen der Überzeitarbeit müssen zumutbar sind und die maximale Wochenarbeitszeit für Büroarbeit von $45 \mathrm{~h}$ muss eingehalten werden (Ausnahmeregelungen für bestimmte Berufsgattungen). Die geleisteten Überstunden können monetär abgegolten werden (Schweizerische Eidgenossenschaft o. J.c.). Für Nacht- und Sonntags- sowie Feiertagsarbeit gelten strengere Regeln. Diese ist zunächst grundsätzlich verboten. Nur bei bewilligten Ausnahmen und bei speziellen Unternehmen sowie Berufsgattungen gibt es eine Sondererlaubnis. Im Falle einer solchen Erlaubnis sind die Unternehmen in besonderem Maße für die Sicherheit und Gesundheit der Mitarbeitenden verantwortlich (Schweizerische Eidgenossenschaft 2015b). Die Arbeitszeiterfassung ist im Arbeitsgesetz (ArG) und den zugehörigen Verordnungen (ArGV) als zwingende Norm des öffentlichen Rechts geregelt (insbesondere Art. 46 ArG und Art. 73 ArGV 1, Art. 73a ArGV 1 und Art. 73b ArGV 1). Die Arbeitszeit beschreibt die tatsächliche zeitliche Beanspruchung des Mitarbeitenden (Arbeitgeberverband Basel 2014). Die Arbeitgeber sind gesetzlich verpflichtet, die Arbeitszeiten der Arbeitnehmer zu erfassen (Schweizerische Eidgenossenschaft 2016). Die Arbeitgeber müssen die geleisteten Arbeitszeiten der Beschäftigten, inklusive Ausgleichs- und Überzeitarbeit, nachweisen können. Ebenfalls müssen die Ruhe- oder Ersatzruhetage sowie die gesetzlich verpflichtenden Pausen dokumentiert werden. Bei Nichteinhaltung der Regelung drohen Strafmaßnahmen, von Verwarnungen zu Bußgeldern bis hin zur Schließung des Unternehmens. Ausgenommen von der Dokumentationspflicht sind lediglich Angestellte des Topmanagements, die an wichtigen Unternehmensentscheidungen beteiligt sind und ihre Arbeitszeit selbstständig organisieren können. Darüber hinaus gibt es seit Januar 2016 zwei zusätzliche Ausnahmeoptionen, um dem zunehmenden Flexibilisierungs- und Mobilitätsbedarf der Wirtschaft entsprechen zu können. Mitarbeitende, die über ein Jahreseinkommen von mehr als CHF 120.000 verfügen, können von der Arbeitszeiterfassung befreit werden (Art. 73a ArGV 1). Dies gilt allerdings nicht für Sonntags- oder Nachtarbeit. Außerdem ist seither neu auch eine partielle oder vereinfachte Zeiterfassung möglich, bei welcher nur die Gesamtdauer der täglichen Arbeitszeit einzutragen ist (Art. 73b ArGV 1) (Schweizerische Eidgenossenschaft 2016). In Verbindung mit Mehrfachbeschäftigung sind Arbeitgeber verpflichtet, die gesamten Arbeits- und Ruhezeiten ihrer Angestellten sicherzustellen, auch wenn diese mehreren Erwerbstätigkeiten nachgehen, was gesetzlich erlaubt ist. Die Nebenerwerbstätigkeit darf allerdings nicht mit dem Geschäft des Hauptarbeitgebers konkurrieren, dem Ansehen des Unternehmens schaden und keine negativen Auswirkungen auf die Haupttätigkeit haben (Treuepflicht der Beschäftigten) (Schweizerische Eidgenossenschaft 2015a). 


\subsection{Implikationen der Digitalisierung für Arbeitsmodelle}

Nachfolgend wird die Fragestellung vertieft, wie die Digitalisierung bestehende Arbeitsmodelle für Büroarbeit beeinflusst und Impulse für neue Arbeitsmodelle geben kann. In dem Zusammenhang werden außerdem mögliche Chancen und Problemstellungen von mit der Digitalisierung einhergehenden Arbeitsmodellen hinterfragt.

In der Literatur wird auch in Verbindung mit der Digitalisierung grundlegend eine Tendenz der Unternehmen zu mehr Flexibilität, Mobilität sowie Autonomie der Beschäftigten ausgemacht. In der Praxis hat sich dies in der Schweiz unter anderem in der Gründung der Work Smart-Initiative durch die Swisscom, die Post, die SBB, die Mobiliar sowie die Firma Witzig im Jahr 2015 niedergeschlagen. Das Ziel dieser Initiative ist es, flexible und ortsunabhängige Arbeitsmodelle in der Schweiz zu fördern. Seit der Gründung haben sich über 150 Schweizer Unternehmen dieser Initiative angeschlossen (Work Smart Initiative 2018). Übergreifende Darstellungen zur digitalisierungsbasierten Veränderung von Arbeitsmodellen sind nur bedingt vorhanden, sodass eine Charakterisierung der Änderungsbewegungen unter Bezugnahme auf die fünf herangezogenen Ausprägungsdimensionen erfolgt. Generell kann aber festgehalten werden, dass heute in großen Unternehmen flexible und agile Arbeitsmodelle bereits eingesetzt und zukünftig vermehrt relevant werden dürften, da eine fortlaufende, fließende Ausrichtung der Arbeitswelten an den Bedürfnissen der Kunden und Mitarbeitenden immer wichtiger wird (Zinser und Boch 2007, S. 57). Alle in den fünf Dimensionen angeführten Arbeitsmodellkomponenten bzw. -varianten sind mehr oder minder von der Digitalisierung betroffen und entwickeln sich im Digitalisierungskontext in Richtung flexiblerer, mobilerer und autonomerer dimensionaler Ausprägungsformen für die bestehenden Arbeitsmodelle oder bringen in Kombination auch neue Arbeitsmodelltypen hervor.

Hinsichtlich der Dimension Arbeitszeit kann festgehalten werden, dass bereits heute für administrative Tätigkeiten Vertrauensarbeitszeit, flexible Arbeitszeitmodelle und amorphe Arbeitszeit in den Unternehmen zunehmend zur Anwendung kommen und von den Mitarbeitenden in der Regel geschätzt werden. Diese Tendenz wird sich in Zukunft vermutlich weiter verstärken (Arbeitgeberverband Basel 2014). Im Hinblick auf die Arbeitsmodelldimension Arbeitsform kann angenommen werden, dass die Arbeitsarten Teilzeitarbeit, Jobsharing und Arbeit auf Abruf weiterhin und vermehrt genutzt werden. Auch diese begünstigen bereits die Flexibilität sowie die Vereinbarkeit von Beruf und Freizeit. Kombinationsmöglichkeiten mit neueren Entwicklungsformen wie Freelancer-Modelle, Human-Cloud-Ansätze sowie mobil-flexibles Arbeiten sind vorstellbar. In Bezug auf die Arbeits-, respektive Personalführung sind Veränderungen bereits vielfach realisiert. So werden Meetings und Gespräche beispielsweise vermehrt ortsungebunden virtuell durchgeführt und Führungskräfte haben bei ihren Führungsentscheidungen oft relevante Informationen in Echtzeit zur Verfügung (Mühlenbeck 2017). In Verbindung mit der Digitalisierung scheinen sich ferner die Erwartungen der Mitarbeitenden an die Führungskräfte stark zu verändern, sodass Unternehmen ihre 
Führungskultur entsprechend hinterfragen und, in Anlehnung an die Erkenntnisse der Studie von Genner et al. (Genner, S. et al. 2017, S. 17), vermehrt in Richtung Selbstführung der Mitarbeitenden, Führung auf räumliche Distanz, identifikations- und zielorientierte Führung, Führung über digitale Kanäle und flache Teamhierarchien anpassen müssen. In Bezug auf die Arbeitsstrukturen zeichnet sich eine zunehmende Entwicklung hin zu agilen Netzwerkstrukturen ab, die durch Selbstorganisation, Vertrauensbasierung, Unternehmertum und ein hohes Maß an Autonomie der Mitarbeitenden gekennzeichnet sind (Sattelberger et al. 2015, S. 272, 276 ff.). Die Ausgestaltung der Büroarbeitsräume wird voraussichtlich im Zuge der Digitalisierung in immer höherem Maß auf mobil-flexibles Arbeiten in Open-Space-Arbeitswelten hin ausgerichtet sein, die der Tendenz Rechnung tragen, dass die in Büros vor Ort verbrachte Zeit der Angestellten sich weiter reduzieren wird. So sind etwa bereits heute selbst im Umfeld traditioneller Arbeitsformen nur maximal $70 \%$ der Büroarbeitsplätze während der Arbeitszeit gleichzeitig besetzt (Zinser und Boch 2007, S. 58). In Verbindung mit den digitalisierungsbedingten und -unterstützten Änderungstendenzen der Arbeitsmodelldimensionen sind auch weitere Änderungen der arbeitsrechtlichen Rahmensetzungen in der Schweiz für die Zukunft zu erwarten. Dem entsprechend hat der Bund 2017 einen Bericht verabschiedet, der die Rahmenbedingungen der Wirtschaft in Hinblick auf die Digitalisierung analysiert. Vor dem Hintergrund der Zielsetzung der Arbeitsplatz- und Wohlstandssicherung wird festgehalten, dass die heutigen Gesetzesgrundlagen zwar weitgehend bereits Spielraum für den Wandel gewährleisten, aber nicht mehr nötige Regulierungen sollen punktuell angepasst und die Rahmenbedingungen für die digitale Arbeitswelt weiter verbessert werden. Hierzu erteilt der Bundesrat Prüfaufträge (Schweizerische Eidgenossenschaft 2018a).

\subsubsection{Entwicklung neuer digitalisierungsbasierter Arbeitsmodelle}

Grundlegende Entwicklungstendenzen für die einzelnen Arbeitsmodelldimensionen wurden unter Bezugnahme auf die bestehende Literatur bereits aufgezeigt. In einem weiteren Konkretisierungsschritt werden exemplarisch mögliche neuere Ausprägungsformen identifiziert, die für sich, oder auch in Kombination mit anderen dimensionalen Ausprägungen, neuartige agile Formen der Zusammenarbeit generieren und in hohem Maße mit den Anforderungen und Möglichkeiten der Digitalisierung kompatibel sind.

Mobil-flexibles Arbeiten steht für eine Kombination verschiedener Arbeitsmerkmale wie etwa Homeoffice, gleitender Arbeitszeit oder Vertrauensarbeitszeit mit räumlich ungebundenem Arbeiten innerhalb oder außerhalb des Unternehmens (Genner et al. 2017, S. 12). Insgesamt $75 \%$ der Schweizer Bevölkerung arbeiten mit steigender Tendenz mittlerweile bereits im Dienstleistungssektor, in dem vorwiegend Wissens- und Kopfarbeit erbracht wird. Von dieser Mehrheit der Beschäftigten arbeiten schon heute fast $30 \%$ mindestens einen halben Tag pro Woche von zu Hause aus (Zobrist und Grampp 2016, S. 10). Es ist davon auszugehen, dass dieser Wert im Zuge der Digitalisierung noch 
deutlich ansteigen wird. Digitalisierung steht dabei auch und insbesondere für den Einsatz mobiler Geräte wie Smartphones, Tablets oder Laptops in Kombination mit Cloudlösungen und VPN-Verbindungen. Hiermit verbunden ist eine wachsende Autonomie der Beschäftigten bei der Art und Weise der Arbeitsdurchführung (Genner et al. 2017, S. 12). Beschäftigte, die in mobil-flexiblen Arbeitsmodellen arbeiten, geben an, dass sie produktiver arbeiten und zufriedener sind, was sich aus Sicht der Unternehmen auch in einer höheren Arbeitsleistung niederschlägt (SBB AG und Swisscom (Schweiz) AG 2013, S. 4).

Freelancer sind freie Mitarbeitende, die ein Honorar für geleistete Dienste erhalten und somit nicht in einem traditionellen Arbeitsverhältnis arbeiten. Zumeist arbeiten Freelancer für verschiedene Auftraggeber. Freelancer sind vor allem in wissensintensiven Berufen tätig, wie etwa als Berater, Informatiker, Grafiker oder Übersetzer (Zobrist und Grampp 2016, S. 7 f.). Gemäß einer repräsentativen Befragung von Deloitte Schweiz und Research Now ist heute bereits jeder vierte Schweizer haupt- oder nebenberuflich als Freelancer tätig (Zobrist und Grampp 2016, S. 8). Der Einsatz von Freelancern ist für Unternehmen mit einer hohen professionellen Flexibilität verbunden, dem entgegen stehen höhere Transaktionskosten und Informationsrisiken. Zobrist und Grampp unterscheiden fünf Arten von Freelancern (Zobrist und Grampp 2016, S. 8 f.):

- Independent Contractor: „Klassische“ Freelancer, die hauptberuflich temporäre und projektbasierte Arbeiten erledigen (ca. $37 \%$ ).

- Moonlighter: Freelancer in Nebentätigkeit, die hauptberuflich einer „traditionellen“ Arbeit nachgehen (ca. $30 \%$ ).

- Diversified Worker: Beschäftigte, die ihr Einkommen mit unterschiedlichen Auftraggebern verdienen (z. B. Teilzeitarbeitnehmerin in einem Unternehmen, die nebenbei für Uber fährt und Online-Englischunterricht gibt) (ca. $23 \%$ ).

- Business Owner: Unternehmer mit bis zu fünf Mitarbeitenden, die sich selbst als Freelancer bezeichnen (ca. $8 \%$ ).

- Temporary Worker: Freelancer, die für einen einzigen Arbeitgeber oder Kunden temporär arbeiten (ca. $2 \%$ ).

Es kann davon ausgegangen werden, dass der Anteil der Freelancer im Sinne einer mit der Digitalisierung einhergehenden weiteren Flexibilisierung der Arbeit in den kommenden Jahren weiter ansteigen wird.

Eng verbunden mit dem Freelancer-Ansatz sind Human Cloud-Arbeitslösungen. Human Cloud-Plattformen wie upwork.com, freelancer.com oder peopleperhour.com zeichnen sich dadurch aus, dass auf ihnen von Arbeitsanbietern und -nachfragern online rund um die Uhr und weltweit Angebote zur Aufgabenwahrnehmung oder für Projektarbeiten platziert werden. Berufliche Tätigkeiten werden in konkrete Aufgaben und separate Projekte aufgeteilt und über die virtuelle Plattform an verschiedene ortsunabhängige selbstständige Arbeitskräfte verteilt, respektive von diesen als befristete Dienstleistungen eingekauft. Im Falle des Erbringens der Dienstleistungen von selbstständig Erwerbstätigen 
sind die einkaufenden Leistungsbezieher nicht verpflichtet, Mindestlöhne und Sozialversicherungsbeiträge zu zahlen (Schwab 2016, S. 73 f.). Für die anbietenden Arbeitskräfte bedeutet diese Form der Arbeitsbeschaffung eine weitgehend autonome Festlegung des Work-Life-Mixes mit größtmöglicher Flexibilität und Mobilität (Schwab 2016, S. 75).

Coaching- und Mentoringansätze der Führung sind sich abzeichnende Antworten auf die geänderten Führungsanforderungen in den flachen Hierarchien zunehmend selbstorganisierter Systeme. In einem solchen Kontext ist die wichtigste Aufgabe von Führungskräften die Schaffung von Verbindlichkeit in flexiblen Netzwerkstrukturen, das Inspirieren und Motivieren ihrer Teams und die kompetenzorientierte Optimierung der Aufgabenzuordnungen zu Personen sowie die systematische Förderung der Kompetenzentwicklung der Mitarbeitenden (Sattelberger et al. 2015, S. 133 f.). Beispiele für eine entsprechende Führungsperspektive sind die kontextabhängigen Ansätze einer systemischen oder symbolischen Führung. Die systemische Führung kommt vor allem bei laufenden Veränderungsprozessen und bei komplexen und unsicherheitsbehafteten Situationen zur Anwendung. Vor diesem Hintergrund führt der Vorgesetzte vor allem indirekt und legitimiert seine Autorität aus der Aufgabe und nicht aus der hierarchischen Position heraus (WEKA 2016). Vorgesetzte agieren hier als Navigatoren in selbstorganisierten Systemen und unterstützen die Mitarbeitenden beim Umgang mit Unsicherheit und Mehrdeutigkeiten. Bei der symbolischen Führung führt der Vorgesetzte über Zeichen, die für bestimmte Verhaltensweisen, Überzeugungen, Selbstbilder und Erwartungen stehen (WEKA 2016). Führen heißt hier vor allem reflektierte Inszenierung gewünschter Verhaltensweisen und Einstellungen durch die Führungskraft über symbolisches Handeln, wodurch auch die kulturellen Rahmensetzungen zum Ausdruck kommen.

In struktureller Hinsicht sehen Sattelberger et al. in Verbindung mit der Digitalisierung für die Zukunft vor allem drei alternative neuartige Organisationstypen, die im Organisationsentwicklungsprozess miteinander in Konkurrenz stehen (Sattelberger et al. 2015, S. 48 ff.). In „flachen Macht-Pyramiden“ kommt die Macht unverändert von oben, und es wird top-down gesteuert, allerdings mit nur wenigen Hierarchieebenen und unterstützt durch dosierte Kulturveränderungen in Verbindung mit den neuen Arbeitsformen. Bei der „Machtverteilung“ liegt ein holokratisches Strukturverständnis zugrunde, das Partizipation und Transparenz in demokratischen und agilen Netzwerkstrukturen favorisiert. Es gibt mehr Teilhabe und Souveränität der Mitarbeitenden, die sich weitgehend selbst führen, sowie Open Innovation. Anstelle der klassischen Strukturen und der hierarchischen Position der Führung werden in holokratischen Organisationsformen „Rollen“ gelebt, die alle Mitarbeitenden innehaben (Meyer 2016). „Machtkonzentration“ steht als letzter hybrider Organisationstypus für eine zweifache Machtkonzentration. Machtbasis ist die Innovation, und die Machtspitze wird durch die strategische und finanzielle Führung repräsentiert. Die operative Handlungskompetenz ist hier über das Empowerment der Mitarbeitenden dezentralisiert, die Steuerung erfolgt jedoch nach feudalistischen Prinzipien. Loyale und transformationsfähige Mitarbeiter, welche als Kernbelegschaft das Wissen des Unternehmens sichern, werden spirituell vereinnahmt. 
Berufliche Karrieren werden entweder über Projekte oder als Expertenkarrieren innerhalb einer offenen Wissenscommunity entwickelt (Sattelberger et al. 2015, S. 48 ff.).

Neuere Ansätze der Büroraumgestaltung tragen diesen strukturellen und führungsbezogenen Veränderungen im physischen Arbeitsumfeld Rechnung. Mobil-flexible Büronetzwerke integrieren die Büro- und Heimarbeitswelten und mobil-flexibles Arbeiten, das Arbeitsinhalte in Projekten strukturiert, wird immer mehr zu einer Normalität, wie sie in vielen Großunternehmen heute schon vorgefunden werden kann (Witzig the office company 2014). In Verbindung mit der aufstrebenden Sharing Economy erfolgt eine Erweiterung der Raumperspektive über das Homeoffice hinaus auf temporär genutzte und angemietete Büroräume. Coworking Spaces sind derzeit im Trend und werden über Plattformanbieter wie LiquidSpace oder ShareDesk vermarktet. Insbesondere Freelancer, Jungunternehmer und Mitarbeitende von Unternehmen nutzen die stundenweise Anmietbarkeit von Büroplätzen oder Meetingräumen in zumeist zentralen City-Lagen immer mehr (Zobrist und Grampp 2016, S. 11). Im Jahr 2017 gab es in der Schweiz bereits an über 180 Standorten auf mehr als 70.000 Quadratmetern Arbeitsfläche rund 6700 Coworking-Arbeitsplätze. Der Median hinsichtlich der Zahl der Arbeitsplätze liegt bei 20, die verfügbare Arbeitsfläche schwankt dabei zwischen 30 und 2500 Quadratmeter. Insbesondere Unternehmen mit mehr als 1000 Mitarbeitenden möchten in den kommenden Jahren unter Flexibilitäts- und Kostengesichtspunkten für ihre Mitarbeitenden solche Coworking Spaces dauerhaft zur Verfügung stellen (Auf der Maur 2017, S. 7 ff.). Ein visionäres Zukunftsbild digitaler Arbeitswelten entwickeln Zinser und Boch (2007, S. 34 f.): In vernetzten Städten sind Büro-, Projekt- und Ideenräume und Think Tanks als intelligente Knotenpunkte präsent, die urbanes Wohnen und Arbeiten integrierbar machen.

\subsubsection{Chancen und Risiken der Digitalisierung für Arbeitsmodelle}

Mit der Digitalisierung und den angeführten komplementären und unterstützenden Entwicklungstendenzen bei der Ausgestaltung von Arbeitsmodellen sind für Unternehmen Chancen und Risiken verbunden.

Auf der Chancenseite neuer flexibler und mobilitätsorientierter Arbeitsmodelle werden aus Sicht der Angestellten vor allem eine höhere persönliche Flexibilität, eine bessere Vereinbarkeit von Familie und Beruf sowie die Reduktion der Arbeitswegzeiten angeführt. Unternehmen profitieren von einer effizienteren Nutzung der Büroflächen, sparen Kosten, können sich als attraktiver Arbeitgeber am Arbeitsmarkt positionieren und haben produktivere und zufriedenere Mitarbeiter. Vermietet das Unternehmen zudem Arbeitsplätze an Externe, können zusätzliche Einnahmen generiert sowie neue Netzwerke erschlossen werden, auch im Hinblick auf die steigende Anzahl an Freelancern (Zobrist und Grampp 2016, S. 16). Generell ermöglicht die Digitalisierung eine erleichterte Flexibilisierung des Arbeitsmarkts (Reynard und Derder 2017, S. 4). Mit den neuen 
Arbeitsmodellen im digitalen Zeitalter gehen auch eine höhere Innovationsfähigkeit und potenzielle Schübe für die Entwicklung neuer Produkte und Dienstleistungen einher (Schweizer Verband der Telekommunikation 2015, S. 20).

Der digitale Fortschritt ermöglicht heute ein Arbeiten, wann und wo immer man dies möchte. Dies hat jedoch aus Sicht der Arbeitnehmenden auch seine Schattenseiten, denn gleichzeitig haben immer mehr Menschen Schwierigkeiten, Arbeit und Privatleben sowie berufliche und familiäre Verpflichtungen in Einklang zu bringen. Dies resultiert in einer zunehmenden psychischen Arbeitsbelastung in Form von Stress, zu der auch das Mehr an Verantwortung und Autonomie beitragen kann. Gemäß der repräsentativen Schweizer Job-Stress-Index-Studie von 2016 ist jeder vierte Erwerbstätige gestresst im Job. Die Ausfälle von Mitarbeitenden aufgrund von Stress kosten die Schweizer Arbeitgeber jährlich ca. 5,7 Mrd. Franken. In welchem Umfang hierbei spezifische Stressfaktoren des digitalen Wandels der Arbeitswelt beteiligt sind, ist allerdings noch unklar und schwer einschätzbar (Genner et al. 2017, S. 39 f.). Die neuen Arbeitsmodellformen können bei den betroffenen Beschäftigten auch Ängste auslösen. Nicht jeder bringt von vorneherein das ideale Soll-Kompetenz- und Persönlichkeitsprofil eines selbstorganisierten, autonom und hochflexibel arbeitenden Experten mit, der sich souverän in agilen Netzwerkstrukturen bewegen kann. Außerdem geht mit der Digitalisierung der Arbeit auch bei vielen die Sorge einher, dass derzeitige menschliche Bürotätigkeiten immer mehr von intelligenten digitalen Systemen ersetzt werden. So sind gemäß der Studie „The Future of Employment" von Frey und Osborne aus dem Jahr 2013 fast 50 \% der derzeitigen Arbeitsplätze dem Risiko einer Eliminierung durch digitale Rationalisierung ausgesetzt (Frey und Osborne 2013). In Verbindung mit der Gestaltung der Rahmenbedingungen neuer Arbeitsformen und -strukturen (z. B. physische und virtuelle Präsenzzeiten und -orte, Arbeits- und Ruhezonenraumgestaltung etc.) besteht bei entsprechenden Defiziten die Gefahr, dass sich bei den Mitarbeitenden ein Identifikations- und Bindungsverlust gegenüber dem Arbeitgeber einstellt und der soziale Austausch mit Kollegen leidet oder gar verloren geht. Die erhöhte Flexibilität und räumliche Ungebundenheit mit globaler Datenverfügbarkeit geht ferner mit nicht unbeträchtlichen Risiken bezüglich der Sicherheit von sensiblen Informationen und Daten einher (Zobrist und Grampp 2016, S. 17). Im Hinblick auf die arbeitsrechtlichen Rahmensetzungen besteht bei einem Ausnutzen rechtlicher Grauzonen (z. B. Mindestlohnthematik) bei der Nutzung von Arbeitsvermittlungsplattformen auf Dauer die Gefahr, dass mit restriktiven Interventionen des Gesetzgebers gerechnet werden muss und gegebenenfalls die Reputation als Arbeitgeber leiden kann.

\subsection{Zwischenfazit der konzeptionellen Analyse}

Digitalisierung steht in Verbindung mit der Arbeitswelt heute insbesondere für digitale Arbeitsprozesse und -inhalte, die Beschleunigung von Arbeit und mobil-flexible Arbeitsformen. Neue Ausprägungsformen von Arbeitsmodellen im Sinne von mehrdimensionalen 
Systemen der Zusammenarbeit können anhand der fünf Dimensionen Arbeitszeit, Arbeitsform, Arbeitsführung, Arbeitsstruktur und Arbeitsraum charakterisiert werden. In Verbindung mit der Digitalisierung weisen diese jeweils für sich oder in Kombination Entwicklungstendenzen in Richtung Flexibilisierung, Individualisierung, Selbstorganisation, Autonomie der Mitarbeitenden, partnerschaftliche systemisch-symbolische Führung, Holokratie, Agilität und Mobilität auf. Bei der Einführung der zukunftsgerichteten Arbeitsmodelle sind die geltenden gesetzlichen Regelungen als Restriktion zu berücksichtigen. Die digitalisierungsbasierten oder -unterstützten Arbeitsmodelle sind für die Unternehmen mit einer Vielzahl an Effizienz-, Effektivitäts- und Innovationspotenzialen verbunden, weisen aber auch eine Reihe von Risiken auf, die mit einer defizitären Einführung und Umsetzung, beziehungsweise fehlender oder falsch gesetzter Rahmenbedingungen verbunden sind. Die konzeptionelle Grundsystematik des Zusammenhangs von Digitalisierung, Arbeitsmodellen und Unternehmenserfolg ist in Abb. 7.1 dargestellt.

In Bezug auf die Einführung und Anwendung modifizierter oder neuer Arbeitsmodelle, die die neuen Möglichkeiten der Digitalisierung nutzen, beziehungsweise auf diesen basieren, gibt es in empirischer Hinsicht noch eine geringe wissenschaftliche Aufbereitung der Thematik. Die nachfolgende deskriptive Analyse des Einsatzes moderner Arbeitsmodelle in Schweizer Großunternehmen setzt an dieser identifizierten Forschungslücke mit der Intention an, Hinweise für Best Practices und Optimierungspotenziale von Arbeitsmodellen in der Praxis abzuleiten.

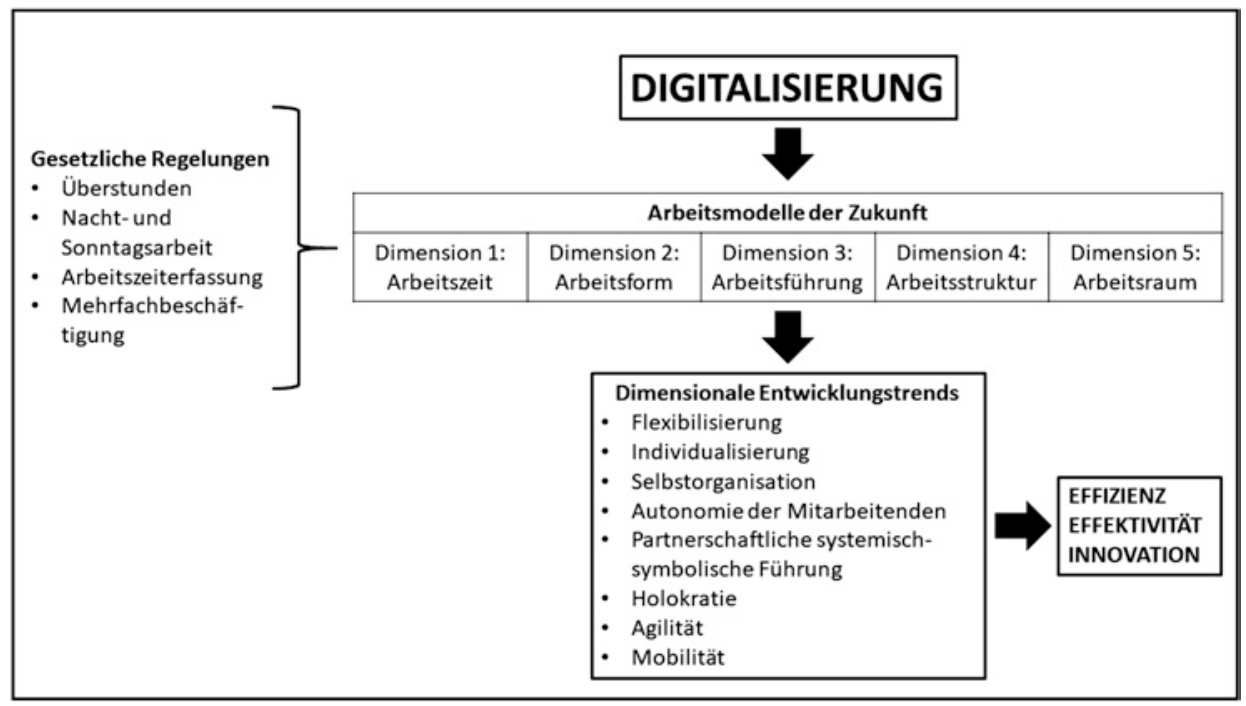

Abb. 7.1 Digitalisierung, Arbeitsmodelle und Unternehmenserfolg. (Eigene Darstellung) 


\subsection{Empirische Untersuchung}

In einem ersten Schritt wird im folgenden Abschnitt zunächst das methodische Vorgehen der empirischen Analyse gekennzeichnet. Es folgen danach eine Darstellung der wichtigsten Ergebnisse der Erhebung und eine abschließende Diskussion.

\subsubsection{Forschungsmethodik}

Bei der Festlegung des Forschungsansatzes wurde das Vorgehen an der Prozessdarstellung von Glogner-Pilz (2012) gemäß Abb. 7.2 ausgerichtet.

Vor dem Hintergrund der interessierenden offenen Fragestellungen wurde kein quantitatives, sondern ein interpretatives qualitatives Vorgehen für die Erhebung gewählt, bei dem das subjektbezogene Verstehen von Zusammenhängen, Erfahrungen und Verhaltensweisen in Bezug auf ausgewählte Gesprächspersonen im Vordergrund steht (Röbken und Wetzel 2016, S. 12 ff.). In Verbindung mit der Fokussierung auf die Realsituation in Großunternehmen wurde ein vergleichender Fallstudienansatz auf der Grundlage leitfadengestützter Experteninterviews als geeignet angesehen.

Insgesamt wurden zehn Interviews in drei Schweizer Großunternehmen durchgeführt, bei denen vermutet werden konnte, dass bereits Erfahrungen bei der Umsetzung von digitalen Veränderungsprozessen und der Anwendung von hiermit verbundenen neuen Arbeitsmodellen bestehen. Selektionskriterien für die Samplebildung, respektive Fallauswahl waren die Klassifizierung als Großunternehmen mit mehr als 250 Mitarbeitenden und Hauptstandort in der Schweiz, als besonders innovativ bekannte Großunternehmen oder als Unternehmen, die die Charta der Work Smart-Initiative (Work Smart Initiative 2018) unterzeichnet haben. Außerdem wurde themenbezogen ein Schwerpunkt auf besonders von der Digitalisierung betroffene kundenorientierte Unternehmen und Organisationen in Dienstleistungsbranchen und der öffentlichen Verwaltung gelegt. Vor diesem Hintergrund konnten 25 potenziell geeignete Unternehmen identifiziert und im März/April 2018 angefragt werden. Fünf der angefragten Unternehmen/Organisationen waren grundsätzlich für eine Mitwirkung an der Studie offen, von den fünf wurden im Hinblick auf die Kriterienerfüllung drei ausgewählt, die alle auch die Charta der Work Smart-Initiative unterzeichnet haben. Konkret handelt es sich bei den ausgewählten Organisationen um die Schweizerische Bundesbahnen (SBB) mit fünf Interviewpartnern, das Bundesamt für Informatik (BIT) mit drei Interviewpartnern und ein namhaftes Versicherungsunternehmen mit zwei Interviewpartnern, das sich anonym an der Studie beteiligt hat. Die Interviews wurden anhand eines Leitfadens im Frühling/Sommer 2018 vor Ort am jeweiligen Arbeitsplatz der Interviewpartner durchgeführt. Der Interviewleitfaden wurde auf Wunsch den Befragten vorgängig zugestellt. Die Interviews wurden zudem alle in Mundart geführt, damit keine sprachlichen Hürden entstanden. Die Befragten gehören verschiedenen Funktionen und Hierarchieebenen an und zeichnen 
Abb. 7.2 Prozessablauf der empirischen Untersuchung. (Eigene Darstellung in Anlehnung an Glogner-Pilz 2012)

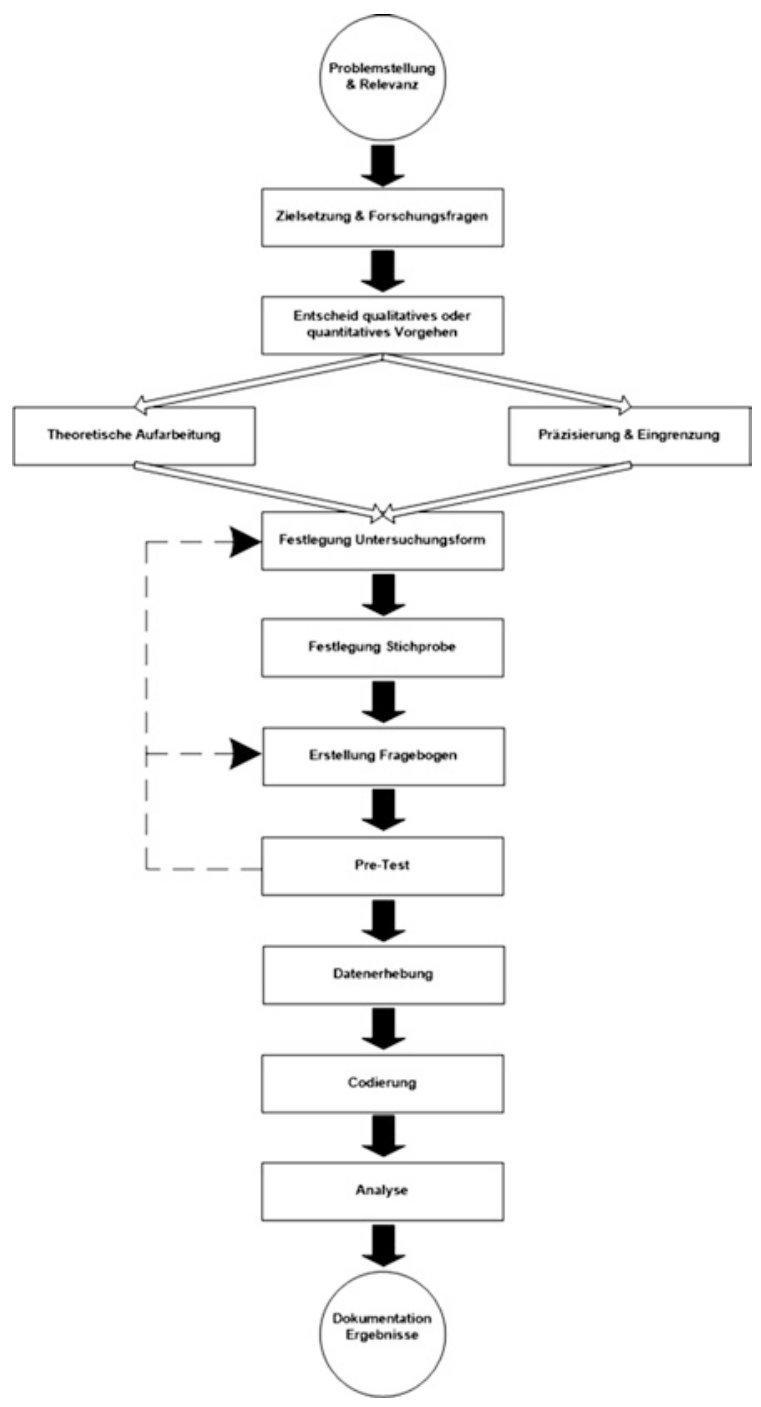

sich alle durch ein Arbeitsumfeld aus, das durch neue Formen der Zusammenarbeit und Entwicklungen im Umfeld der Digitalisierung geprägt ist (Human Resource Management, IT-Abteilungen, Organisationsentwicklung, Projektmanagement). Die Breite der Profile soll eine Abdeckung verschiedener Aspekte der Thematik Digitalisierung und Arbeitsmodelle gewährleisten.

Für die Interviews wurde ein leitfadengestützter Fragebogen mit sieben Hauptforschungsfragen und achtundzwanzig Subfragen erarbeitet. Die Fragen wurden bewusst sehr offen formuliert, um die Thematik nicht zu sehr konzeptionsgeleitet vorzustrukturieren und Raum für ungelenkte subjektive Praxisperspektiven zu schaffen. Der 
Interviewleitfaden wurde im Rahmen eines Pre-Tests mit mehreren neutralen Personen geprüft und angepasst.

Die durchgeführten und aufgezeichneten Interviews wurden anschließend transkribiert. Die Transkripte wurden danach in Anlehnung an die Themenstruktur des Fragebogens codiert und mittels der Analyse-Software ATLAS.TI ausgewertet.

\subsubsection{Ergebnisse}

Die Ergebnisse werden themenfeldbezogen zunächst anhand fallstudienübergreifender Konsensaussagen der Interviewten vorgestellt und dann fallbezogen weiter konkretisiert.

\subsubsection{Bedeutung und Auswirkungen der Digitalisierung für Unternehmen und Mitarbeitende}

Die Breite der begrifflichen Festlegung dessen, was „Digitalisierung“ inhaltlich bedeutet, hat sich auch bei den Experteninterviews gezeigt. Alle Teilnehmenden der Befragung sehen die Aktualität und Relevanz des Themas und verbinden dieses vor allem mit der Effizienzsteigerung elektronischer Prozesse und neuer Geschäftsmodelle. Vereinzelt wird der Begriff auch mit „Automatisierung“ gleichgesetzt. Die hohe Bedeutung der Digitalisierung für auf alle Bereiche der Gesellschaft und für die menschliche Arbeit wird von allen Befragten wahrgenommen (BIT (P6, P7, P9) 2018; SBB (P1, P2, P3, P5, P10) 2018; Versicherungsunternehmen (P4, P8) 2018). Die Befragten der SBB betrachten darüber hinaus die Digitalisierung zudem als Synonym für die papierlose Vernetzung der Daten und Einheiten mit Hard- und Software und sehen mit ihr auch eine bestimmte Arbeitskultur und Denkhaltung für digitale Veränderungen und den Umgang mit wachsender Volatilität, Unsicherheit, Komplexität und Mehrdeutigkeiten verbunden (SBB (P1, P2, P3, P5, P10) 2018). Beim BIT wurde ferner auf einen definitorischen Ansatz der Universität St. Gallen Bezug genommen: „Unter digitaler Transformation verstehen wir die Kombination von Veränderungen in Strategie, Geschäftsmodellen, Organisationsprozessen und -kulturen in Unternehmen durch Einsatz von digitalen Technologien mit dem Ziel, die Wettbewerbsfähigkeit zu steigern“"(BIT (P6) 2018, Z. 12 f.). Ergänzend hierzu wird im BIT im Rahmen der Digitalisierung ein vermehrter Einsatz von Maschinen mit intelligenten Programmen und mit künstlicher Intelligenz verknüpft (BIT (P6, P7, P9) 2018). Im Versicherungsunternehmen wird in einem engeren Verständnis Digitalisierung mit der Zurverfügungstellung digitaler Daten gleichgesetzt und in einem weiteren Sinne eine digitale Prozessgestaltung und -programmierung sowie ein hohes Maß von Automatisierung als wesentlich betrachtet (Versicherungsunternehmen (P4, P8) 2018).

Die Auswirkungen der Digitalisierung auf die Unternehmen und deren Mitarbeitende sind untrennbar miteinander verbunden. Alle Befragten sehen eine generelle Beschleunigung der Aktivitäten und gestiegene Erwartungshaltungen der Kunden, die durch das Internet eine wachsende Markttransparenz in Bezug auf die Qualität und Preise der Produkte und Dienstleistungen für sich nutzen. Dies führt insgesamt zu 
einem höheren Konkurrenzdruck (BIT (P6, P7, P9) 2018; SBB (P1, P2, P3, P5, P10) 2018; Versicherungsunternehmen (P4, P8) 2018). Bei der SBB wird die Digitalisierung ferner als vergleichbar mit anderen Veränderungen wahrgenommen: „Es wird Sachen geben, welche es zukünftig nicht mehr geben wird, aber es wird auch Neues entstehen, wovon wir aktuell noch gar nicht wissen, dass wir es einmal brauchen werden“ (SBB (P1) 2018, Z. 286 f.). Insgesamt sei durch die Digitalisierung heute schon die Zusammenarbeit anders geworden und die Kommunikation über digitale Kanäle habe zugenommen. Mit dem „Working Anywhere“ hat die SBB ein Arbeitsmodell eingeführt, das es den Mitarbeitenden ermöglicht, ortsunabhängig und flexibel zu arbeiten, sodass auch die Züge entlastet werden. Wichtig bei der Digitalisierung sei, sich zu fragen, wohin man wolle, sich auf die wesentlichen Fragestellungen zu konzentrieren und dann die erforderlichen Ressourcen einzusetzen (SBB (P1, P2, P3, P5, P10) 2018). Das Versicherungsunternehmen arbeitet mit einem gleichgerichteten Modellansatz „Work Anywhere, Anytime and Smart“. Darüber hinaus wurde die Entwicklungsabteilung „Unit Development" initialisierend komplett digitalisiert. Aus Sicht der Befragten des Versicherungsunternehmens ist die Digitalisierung die nächste ,industrielle Revolution“. Sie habe die gleichen Auswirkungen wie die Erfindung der Dampfmaschine. Dank der Digitalisierung würden nicht nur neue Jobprofile entstehen, sondern auch ergänzende neue Kommunikationskanäle zwischen Mitarbeitenden und Kunden: „Kunden nutzen andere Zugänge; über WhatsApp werden Kundenberater heute kontaktiert, was noch vor ein paar Jahren undenkbar gewesen wäre“ (Versicherungsunternehmen (P4) 2018, Z. 14 f.). Ferner würden durch die Digitalisierung auch neue Geschäftsmodelle ermöglicht, die die Konkurrenzfähigkeit auch in Zukunft gewährleisten sollen (Versicherungsunternehmen (P4, P8) 2018). Aus Sicht der Befragten des BIT verändert die Digitalisierung das Zusammenarbeiten und das Führen von Mitarbeitenden nachhaltig: Die Freiheitsgrade werden größer, es entstehen neue Arbeitsformen und übergreifende Tätigkeiten und die Orientierung an individuellen Bedürfnissen wird begünstigt. Außerdem verbessere sich der Umgang mit Unsicherheit und Ungewissheit. Hier stehe man bei den Digitalisierungsauswirkungen allerdings erst am Anfang einer weitgehenden Veränderung (BIT (P6, P7, P9) 2018).

\subsubsection{Aktuelle Arbeitsmodelle - Ausprägungsformen und Erfolgswirksamkeit}

Die Frage danach, welche aktuellen Arbeitsmodelle derzeit bei den untersuchten Großunternehmen/-organisationen zum Einsatz kommen, wurde zunächst von den Teilnehmenden mit Bezug auf die Arbeitszeitgestaltung hin beantwortetet. Sie verwiesen durchgängig auf vorhandene Jahresarbeitszeit-, Vertrauensarbeitszeit- und Gleitzeitmodelle. Obwohl die untersuchten Organisationen somit alle im Grundsatz eine flexible Arbeitsgestaltung ermöglichen, gibt es in verschiedenen Bereichen und für bestimmte Arbeitstätigkeiten nach wie vor noch klassische Arbeitsmodelle (BIT (P6, P7, P9) 2018; SBB (P1, P2, P3, P5, P10) 2018; Versicherungsunternehmen (P4, P8) 2018). So sind beispielsweise bei der SBB Nichtbürotätigkeiten im Betrieb vielfach noch traditionell 
hierarchisch organisiert. Andere Bereiche wie IT oder HR arbeiten stattdessen mit agileren Arbeitsformen. In der HR-Abteilung der SBB wurde beispielsweise vor kurzem „Holacracy“ als Strukturmodell eingeführt. Charakteristisch für solche agilen Arbeitsmodelle bei der SBB seien Teamorientierung, Selbstorganisation, eine erhöhte Autonomie und eine geringere Anzahl an Führungsstufen, respektive flache Hierarchien. Die agilen Arbeitsformen seien leistungs-, output-, und zusammenarbeitsorientiert. Im Weiteren habe man weniger Präsenzzeiten und Homeoffice sei in Absprache mit dem/der Vorgesetzten erlaubt. Auch flexible Pensionierungen sind in der SBB möglich. So kann man etwa ein Zeitguthaben ansparen und in einem gewissen Umfang selber entscheiden, wann man in den Ruhestand eintritt (SBB (P1, P2, P3, P5, P10) 2018). Beim Versicherungsunternehmen gibt es eine klassische „Run-the-Business“-Arbeitsperspektive und agilere, projektbasierte „Change-the-Business“-Arbeitsweisen. Das Versicherungsunternehmen arbeitet in Projekten vor allem mit der agilen Methode „Scrum“ und der Anwendung der ,SAFe“-Logik (Scaled Agile Framework), anhand derer Umsetzungsvorhaben vorgängig gesammelt, priorisiert, unternehmensweit abgestimmt und den Teams zugeteilt werden. Ziel hierbei ist es, die Vorhaben sinnvoll zu bündeln und Konflikte zwischen den verschiedenen Schnittstellen zu senken. Es kommen dabei stabile interdisziplinäre und autonome Teams zum Einsatz (Versicherungsunternehmen (P4, P8) 2018). Das BIT verwendet verschiedene Arbeitsmodelle, die zu den jeweiligen Lebenssituationen und -abschnitten der Mitarbeitenden passen. Für Einsteiger bietet das BIT einerseits die Lehre, und andererseits auch ein praxisintegriertes Bachelorstudium oder ein Berufseinsteiger-Programm für Hochschulpraktikanten an. Für Mitarbeitende, welche eine Familie gründen wollen oder diese bereits haben, besteht die Möglichkeit, in Teilzeit oder Jobsharing zu arbeiten. Für ältere Personen können „Bogenkarrieren“ derart umgesetzt werden, dass Führungspersonen Verantwortung abgeben, und stattdessen eine Beratungsfunktion übernehmen. Ferner sind auch frühzeitige Pensionierungen oder Teilpensionierungen möglich. Darüber hinaus haben Mitarbeitende des BIT die Möglichkeit, Überzeiten für ein Sabbatical-Konto anzusammeln oder sich diese auszahlen zu lassen. Im BIT besteht ferner die Option, „Remote Work“ im Sinne eines orts- und zeitunabhängigen Arbeitens zu praktizieren. Mitarbeitende im BIT haben nicht automatisch ein Anrecht auf Homeoffice, sondern dies wird fallweise betrachtet. Beim BIT ist die Jahresarbeitszeit das dominante Modell für alle Mitarbeitenden. Bei Führungskräften kommt die Vertrauensarbeitszeit zum Einsatz. Die entsprechenden Festlegungen können aber personen- und teamabhängig variieren. Da das BIT aufgabenbedingt über arbeitszeitbezogene Sonderbewilligungen des Bundes verfügt, stellt flexibles Arbeiten beispielsweise auch an Wochenenden kaum ein Problem dar (BIT (P6, P7, P9) 2018).

Hinsichtlich der Fragestellung, ob und in welcher Form sich die bisherigen Arbeitsmodelle bewährt haben, wurde seitens der Befragten der SBB darauf verwiesen, dass die Erfolgswirksamkeit der eingesetzten Modelle eine Sache der Gewohnheit, Einfachheit und der sozialen Normierung sei. Arbeitsmodelle müssten zur aktuellen Entwicklung und der gegenwärtigen Unternehmenssituation passen. Deshalb gäbe es bei der SBB Arbeitsmodelle, die früher schon funktioniert haben und auch heute noch funktionieren 
würden. Die kontinuierliche Fortführung und Weiterentwicklung von Arbeitsmodellen bringe Stabilität und Sicherheit (SBB (P1, P2, P3, P5, P10) 2018). In eine ähnliche Richtung geht die Einschätzung der BIT-Teilnehmenden. Die praktizierten BIT-Arbeitsmodelle hätten bislang die Bedürfnisse der einzelnen Mitarbeitenden gut abdecken können und seien an der Individualisierung und Flexibilisierung der Arbeit ausgerichtet. Dies fördere auch die Zufriedenheit der Mitarbeitenden (BIT (P6, P7, P9) 2018). Beim Versicherungsunternehmen gibt es Arbeitsmodelle, die sich mehr bewährt und solche, die sich weniger bewährt haben. Besonders bewährt hätten sich diejenigen Arbeitsmodelle, die effizienzorientiert seien. In einem explorativen oder Projektumfeld hätten sich insbesondere die flexiblen Arbeitsmodelle bewährt (Versicherungsunternehmen (P4, P8) 2018).

\subsubsection{Chancen und Problemfelder für bestehende Arbeitsmodelle}

Alle Befragten waren sich einig darüber, dass mit der Digitalisierung und der damit einhergehenden Möglichkeiten der Arbeitsmodellgestaltung viele Vorteile und Chancen verbunden seien. Eine bessere Vereinbarung von Beruf und Privatleben wurde bei allen Fallstudienteilnehmenden als positiver Effekt genannt. Auch die gestiegene Flexibilität sowie orts- und zeitunabhängiges Arbeiten sehen alle als Vorteil für das Unternehmen/ die Organisation und die Mitarbeitenden. Außerdem werden für die Mitarbeitenden mehr Möglichkeiten zur Übernahme von Verantwortung, zur Veränderung und zur Erweiterung des eigenen Horizonts durch interdisziplinäres Arbeiten und Wissensaustausch angeführt. Die Befragten sehen vor allem die flexiblen Arbeitsmodelle als wichtigen Faktor, um sich am Arbeitsmarkt als attraktiver Arbeitgeber positionieren zu können. Als nachteilig in Verbindung mit den praktizierten modernen Arbeitsmodellen erachten die Befragungsteilnehmenden, dass die Mitarbeitenden durch erhöhte Anforderungen und mehr Verantwortung auch überfordert oder psychisch überlastet werden können. Zudem werde der persönliche Kontakt durch die digitalen technologischen Möglichkeiten geringer (BIT (P6, P7, P9) 2018; SBB (P1, P2, P3, P5, P10) 2018; Versicherungsunternehmen (P4, P8) 2018). Bei der SBB werden agile Arbeitsmodelle auch mit Einsparungen an Büroflächen in Verbindung gebracht. Viele Mitarbeitende würden von zu Hause aus oder an anderen Orten außerhalb des Büros arbeiten. Für die Arbeitnehmer reduziere sich mit der flexiblen Arbeitsgestaltung vielfach der Stress, da sie auch private Termine wahrnehmen könnten und nicht fix im Büro bleiben müssten bis alle Pendenzen erledigt seien. Im Kontext der Digitalisierung wurden bei der SBB eine Reihe von Projekten zur Arbeitsmodell(weiter)entwicklung gestartet. Ein exemplarisches Projekt ist „MEET“ das zum Ziel hat, Mitarbeitende aller Hierarchieebenen miteinander digital zu verbinden. Arbeitsrelevante Informationen sollen durch gemeinsame digitale Ablagen (eSpace) einfacher für alle zugänglich gemacht werden. Die Mitarbeitenden sollen dadurch ihr Wissen verbreitern und weniger auf direkte Information durch Vorgesetzte angewiesen sein, die dadurch wiederum entlastet werden und sich stärker um ihre Mitarbeitenden und die Personalführung kümmern können (SBB (P1, P2, P3, P5, P10) 2018). Die Befragten des Versicherungsunternehmens sehen durch die angewandten modernen Arbeitsmodelle 
eine Beschleunigung und Effizienzsteigerung bei den Prozessen. Außerdem würden komplexe Aufgaben in interdisziplinären Teams effektiver bearbeitet und seien stärker an den Kundenbedürfnissen ausgerichtet. In diesem Zusammenhang sei auch die Vielfalt der gelebten Arbeitsmodelle im Vergleich zu früher größer geworden (Versicherungsunternehmen (P4, P8) 2018). Im BIT werden die Erweiterungen der Arbeitsmodelle als Reaktion auf die gestiegenen Anforderungen der Mitarbeitenden an die Arbeitgeber wahrgenommen. Hieraus seien neue Rollen geschaffen worden und das Führungsverhalten habe sich stärker in Richtung selbstorganisierter Teams entwickelt. Mitarbeitende würden dadurch autonomer und flexibler arbeiten, was wiederum deren Motivation steigere. Ein stärkeres Involvement der Kunden in Arbeits- und Entwicklungsprozesse trage zur Verbesserung der Kundenzufriedenheit bei (BIT (P6, P7, P9) 2018).

Von den Befragten der SBB wird neben einer möglichen psychischen Überlastung von Mitarbeitenden durch die veränderten Arbeitsweisen wahrgenommen, dass es auch Mitarbeitende gibt, die die avisierte Flexibilität und Autonomie nicht wollen und lieber in stabilen hierarchischen Strukturen arbeiten. Gründe hierfür könnten Angst, Unsicherheit oder der Wunsch nach klaren Aufträgen oder Arbeitsleitplanken sein. Auch die Angst vor Arbeitsverlust oder einer unerwünschten Änderung der Arbeitstätigkeit spielten hierbei eine Rolle. So würden etwa zunehmend die bisherigen Arbeitstätigkeiten von Maschinen/Systemen ausgeführt und vom Menschen nur noch kontrolliert. Probleme in Verbindung mit der Digitalisierung und veränderten Arbeitswelten würden sich vor allem dann einstellen, wenn einzelne Menschen noch nicht in der Lage seien, mit der Digitalisierung und deren Auswirkungen auf ihre Arbeit umzugehen, denn „es hat noch keine Transformation gegeben, bei welcher alle mitgenommen wurden“ (SBB P2 2018, Z. 67 f.). Auch für die Vorgesetzten ändere sich die Führung: Sie müssten Macht an die Mitarbeitenden abgeben und sich aufgrund der erhöhten Flexibilität und Mobilität verstärkt mit den Mitarbeitenden abstimmen. Die Abstimmungen erfolgten dabei vermehrt nicht mehr persönlich, sondern digital. Man sitze nicht mehr im gleichen Raum und könne daher auch Mimik, Gestik, Stimmung und Atmosphäre schwerer wahrnehmen (SBB (P1, P2, P3, P5, P10) 2018). Für die Studienteilnehmenden des Versicherungsunternehmens sind mit den neuen Arbeitsmodellen im Digitalisierungskontext auch Unklarheiten bezüglich der Rollenverteilung verbunden. Ferner gingen durch die wachsende Agilität zunehmend Strukturen und Stabilität im Arbeitsalltag verloren. Mit den heutigen Arbeitsmodellen stoße man an operative Grenzen, da immer mehr Mitarbeitende agil und in Projekten arbeiten möchten, was etwa eine Zielplanung und -vereinbarung erschwere (Versicherungsunternehmen (P4, P8) 2018). Die Befragten des BIT sehen einen weiteren Nachteil der neuen Arbeitsmodellvarianten darin, dass feste Arbeitszeiten und der bis anhin fixe Arbeitsort nun zu einer Abmachungssache geworden seien. Man könne nicht mehr erwarten, die Kollegen im Büro automatisch anzutreffen, sondern müsse Termine im Vorfeld planen. Ferner sei das hohe Maß der eigenen Variabilität und Flexibilität bei der Zusammenarbeit mit Partnerunternehmen und -organisationen oftmals schwierig für die Zusammenarbeit, da nicht alle auf einem vergleichbaren Agilitätsniveau unterwegs seien (BIT (P6, P7, P9) 2018). 


\subsubsection{Treiber für die Veränderung der Arbeitsmodelle}

Die wichtigsten Auslöser und Triebkräfte für die Weiter- und Neuentwicklung von Arbeitsmodellen sind bei allen untersuchten Organisationen mehrheitlich entweder nur auf Leitungsebene (top-down) oder in einem Leitungs-Basis-Mix (top-down - bottom-up) positioniert. Ausgangspunkt für Anpassungen sind (digitalisierungsbedingte) Veränderungen des Umfeldes, wie die Kundenanforderungen, die Bedürfnisse der Mitarbeitenden und die technologischen und kulturellen Entwicklungen sowie die Globalisierung und die Entwicklung neuer Medien. Externe Beratungsunternehmen spielen dabei bei den befragten Organisationen bislang keine Rolle, denn es sei schwierig von außen unternehmensspezifisch geeignete Arbeitsmodelle zu bestimmen (BIT (P6, P7, P9) 2018; SBB (P1, P2, P3, P5, P10) 2018; Versicherungsunternehmen (P4, P8) 2018). Bei der SBB wurden in Bezug auf den Anstoß und das Voranbringen von Arbeitsmodellanpassungen sowohl Top-down-, als auch Bottom-up- oder integrierte Vorgehensweisen ausgemacht. Im besten Falle kämen Impulse für Anpassungen direkt und selbstorganisiert aus den Teams heraus, was jedoch ein starkes Miteinander und eine hohe intrinsische Motivation der Teammitglieder voraussetze. Oftmals brauche es aber einen „Fahnenträger“, der die Digitalisierung und die Anpassung der Arbeitsmodelle von innen nach außen vorantreibe. Angesichts der Unternehmensgröße seien im Thema aber auch Top-Down-Prozesse erforderlich, um zu vermeiden, dass nicht jeder Bereich abgekoppelt für sich etwas erarbeite (SBB (P1, P2, P3, P5, P10) 2018). Beim Versicherungsunternehmen kommt der Anstoß für Veränderungen aus dem HR-Bereich und vom CEO. Besonders wichtig dabei sei, dass man den Mitarbeitenden erkläre, warum die Anpassungen vorgenommen werden müssten und was deren Nutzen sei (Versicherungsunternehmen (P4, P8) 2018). Auch beim BIT wird die Digitalisierung und Entwicklung der Arbeitsmodelle primär von der Geschäftsleitung in Verbindung mit den veränderten Geschäftsanforderungen der Kunden vorangetrieben. Derzeit steht dabei der Prozessverbesserungsansatz „DevOps“ im Mittelpunkt, der das Zusammenwachsen von Entwicklung, Engineering und Betrieb zum Gegenstand hat. Mit DevOps einher geht ein Arbeitsmodell, das den End-to-End-Aspekt fokussiert. Ein DevOps-Team bearbeitet dabei ein Projekt oder einen Kundenauftrag von Anfang bis Ende. Das Team setzt sich aus verschiedenen Mitarbeitenden verschiedener Bereiche zusammen, die über die benötigten Skills verfügen. Ein wichtiger externer Treiber für die Weiterentwicklung der Arbeitsmodelle sind beim BIT auch die massiven Engpässe am Arbeitsmarkt für hochqualifizierte Arbeitskräfte und Talente. Dies erfordere zwingend eine Positionierung als attraktiver zukunftsorientierter Arbeitgeber. In diesem Zusammenhang wurde eine strategische Initiative durch die Geschäftsleitung auf den Weg gebracht, die eine Analyse der Rahmenbedingungen zum Gegenstand hat, um zukünftige Arbeitsmodelle und attraktive Arbeitsbedingungen zu fundieren (BIT (P6, P7, P9) 2018).

\subsubsection{Vorgehen bei der Einführung und Anpassung von Arbeitsmodellen}

Keines der untersuchten Unternehmen arbeitet mit spezifischen und stark standardisierten Verfahren und Prozessen für die Wahl und Einführung neuer Arbeitsmodelle, 
sondern greift auf geläufige interne Projektmethoden zurück. Ein „One-size-fits-all“-Arbeitsmodell gibt es ebenfalls nicht. Wichtig für die erfolgreiche Implementierung von Arbeitsmodellen sei es, dass diese zum Unternehmen, zu den Mitarbeitenden, zur Aufgabenstellung sowie zur Kultur passten, die Bedürfnisse abdeckten und für die Organisation einen Mehrwert generierten. Nur so könne das Arbeitsmodell erfolgreich werden. Die Grundstruktur des Vorgehens bei der Einführung oder Anpassung von Arbeitsmodellen ist jedoch bei allen Organisationen vergleichbar. In der Regel werden zunächst neue Bedürfnisse und Anforderungen abgeklärt. Anschließend werden die betroffenen Personen so weit wie möglich zu Beteiligten gemacht, und es wird gemeinsam schrittweise und mit Pilotumsetzungen an einer Lösung gearbeitet (BIT (P6, P7, P9) 2018; SBB (P1, P2, P3, P5, P10) 2018; Versicherungsunternehmen (P4, P8) 2018). Bei der SBB werden üblicherweise grundlegende Veränderungen in Form von Projekten umgesetzt. Bei der Auswahl und Einführung neuer Arbeitsmodelle werden unterschiedliche betroffene Personen und Bereiche eingebunden (z. B. HR, Immobilien, Infrastruktur etc.). Beispielhaft wurde das interne Projekt „BYTE“ für die Arbeitsplatzgestaltung des neuen SBB-Gebäudes in Bern Wankdorf angeführt. Für die Betrachtung des Arbeitsumfeldes wurden Workshops mit den beteiligten Personen durchgeführt, um deren Bedürfnisse abzuholen und gemeinsam Gestaltungsmöglichkeiten zu erarbeiten. Der hohe Partizipationsgrad benötigte zwar Zeit und Ressourcen, hat sich aber gelohnt. Eine weitere Entscheidungsgrundlage waren Beobachtungen im Arbeitsumfeld und Befragungen der Mitarbeitenden mittels Fragebogen. Bei den Reaktionen in den ersten Umsetzungstagen zeigte sich wie gut die neuen Arbeitsstrukturen und Raumkonzepte ankamen, und wo noch Verbesserungen erforderlich waren (SBB (P1, P2, P3, P5, P10) 2018). Beim Versicherungsunternehmen kommt bei Aufgabenstellungen wie der Einführung oder Anpassung von Arbeitsmodellen ein Reifemodell zum Einsatz, das eine Phasenstrukturierung zugrunde legt und eine kontinuierliche kommunikative Begleitung des Veränderungsprozesses vorsieht. Vorgängig muss dabei Sinn und Zweck der Anpassung eingehend geklärt werden (Versicherungsunternehmen (P4, P8) 2018). Das BIT hebt bei Arbeitsmodellanpassungen und -einführungen auf experimentelle Pilotierungen ab. Ist die Pilotierung erfolgreich, wird das Modell breiter im Unternehmen kommuniziert und top-down ausgerollt. Der Einführungsprozess ist in einen gremiengestützten Entscheidungsrahmen eingebettet, in dem Vertreter aus den HR- und Finanzbereichen die Wirtschaftlichkeit veränderter Arbeitsmodelle mittels KostenNutzen-Betrachtungen analysieren und die grundsätzliche Realisierbarkeit des Veränderungsprojektes prüfen (BIT (P6, P7, P9) 2018).

Ein wichtiger Erfolgsfaktor der Arbeitsmodelleinführung und -weiterentwicklung, der sich bei den Interviews durchgängig herauskristallisiert hat, ist das Einbeziehen unterschiedlicher Stakeholder im Einführungsprozess. Eine weitere wichtige Erfolgskomponente scheint die rechtzeitige Qualifizierung der Mitarbeitenden im Hinblick auf die neuen Arbeitsanforderungen hinsichtlich Agilität und dem Mehr an Verantwortung zu sein. Wichtig bei der Einführung von neuen Arbeitsmodellen ist ferner, dass diese auch nach der Einführung begleitet werden, um ein Rückfallen in alte Verhaltensmuster 
$\mathrm{zu}$ vermeiden. Es braucht ferner eine nachvollziehbare Storyline und eine logische Erklärung für die Veränderung für alle Betroffenen (insbesondere Kundennutzen, Nutzen für die Mitarbeitenden, bessere Zusammenarbeit und Effizienz) (BIT (P6, P7, P9) 2018; SBB (P1, P2, P3, P5, P10) 2018; Versicherungsunternehmen (P4, P8) 2018).

Als größte Hürde für Neueinführungen und Anpassungen von Arbeitsmodellen wurde von allen Interviewten der Faktor Mensch angeführt. Personen, die die neue Arbeitswelt nicht wollen und sich in ihr nicht zurechtfinden, stellen eine zentrale Herausforderung dar. Wenn Arbeitsmodelle angepasst werden, gehe es zwar einerseits um Organisationsentwicklung, vor allem aber auch um die Persönlichkeitsentwicklung der Betroffenen: „Der kleine Haken, den diese Arbeitsformen haben, ist, dass wenn man sie konsequent umsetzen möchte, sie etwas mit Persönlichkeitsentwicklung zu tun haben, nicht mit Personalentwicklung. Und diese Persönlichkeitsentwicklung ist meistens schmerzhafter. Weil ich mich nicht mit meinen Fähigkeiten und Kompetenzen auseinandersetzen muss, sondern ich muss mich mit mir und meiner Person auseinandersetzen." (SBB (P2) 2018, Z. 86 ff.). Es wird immer Personen geben, die mit der neuen Arbeitsweltgestaltung nicht einverstanden sind. Es gibt Personen, die blockieren oder gar sabotieren. Der nachvollziehbare Grund hierfür sind überwiegend jahrelang etablierte Gewohnheiten im früheren Arbeitsumfeld. Die Widerstände müssen akzeptiert und konstruktiv angegangen werden und die Mitarbeitenden sind auf ihrem Weg mit Überzeugungsarbeit und Qualifizierungsmaßnahmen zu begleiten (SBB (P1, P2, P3, P5, P10) 2018; Versicherungsunternehmen (P4, P8) 2018).

\subsubsection{Rahmenbedingungen der Arbeitsmodelle}

Die gesetzlichen Rahmenbedingungen spielen bei allen untersuchten Organisationen in Verbindung mit den angewandten und zukünftigen Arbeitsmodellen eine große Rolle. Auf deren Einhaltung wird konsequent geachtet. Es sind sich alle Befragten einig darüber, dass die heutigen gesetzlichen Rahmenbedingungen bis $\mathrm{zu}$ einem gewissen Grad Flexibilität zulassen, aber in einigen Bereichen an ihre Grenzen stoßen und auf die erhöhten Flexibilitätsanforderungen der Zukunft hin angepasst werden müssen. Anpassungen seien im Hinblick auf die weitere Flexibilisierung von Arbeitszeiten und Leistungserbringungen vor allem beim Schutz der Mitarbeitenden, hinsichtlich Leistungsmessungen und hinsichtlich der Entlohnung nötig (BIT (P6, P7, P9) 2018; SBB (P1, P2, P3, P5, P10) 2018; Versicherungsunternehmen (P4, P8) 2018). Aus Sicht der Befragten der SBB sind die gesetzlichen und internen Regelungen noch wichtiger als früher, weil durch die Digitalisierung frühere Rahmensetzungen wegfallen und neu entwickelnde Grauzonen klare Leitplankensetzungen erforderlich machen. Heutige Rahmenbedingungen funktionieren größtenteils noch, sind aber durch die Ausrichtung auf traditionellere Arbeitsmodelle teilweise intransparent und unfair und weisen Anpassungsbedarfe auf. Dazu gehöre zum Beispiel die Handhabung von Nachtund Sonntagsarbeit, da aufgrund der Autonomie und Flexibilität die Mitarbeitenden manchmal von sich aus abends und an Wochenenden arbeiten und dafür andere Tage frei nehmen. Daraus resultiere etwa die Fragestellung, ob solche Arbeiten dann auch 
zulagenberechtigt sind. Für das Human Resource Management ist es zudem wichtig, dass der Arbeitnehmerschutz (z. B. Ruhezeiten) auch für die neuen flexiblen Arbeitsmodelle gewährleistet bleibt, da eine führungsseitige Kontrolle nur mehr bedingt oder gar nicht mehr gegeben ist. Rahmenbedingungen sollten in dem Zusammenhang eine möglichst klare Trennung von Arbeitszeit und Freizeit ermöglichen (SBB (P1, P2, P3, P5, P10) 2018). Ein weiteres defizitäres Regelungsfeld im Hinblick auf aktuelle und zukünftige neue Arbeitsmodelle ist die Flexibilisierung von Lebensabschnitten während der Erwerbszeit. Wenn man in der Phase der Familiengründung ist, sollte man beispielsweise mehr Zeit für die Familie haben können. Weitere Regelungslücken sind die fehlende Förderung von Jobsharing im Topmanagement oder die Teilzeitarbeit von Mann und Frau. Seitens einigen Befragten wurde auch auf Regelungsdefizite beim Datenschutz im Kontext der Zielsetzung einer jederzeitigen Verfügbarkeit von Unternehmensdaten für einen großen Personenkreis hingewiesen (SBB (P1, P2, P3, P5, P10) 2018; Versicherungsunternehmen (P4, P8) 2018). Seitens der Befragten des BIT werden die Rahmensetzungen für die Auftragsvergaben an Unternehmen oder an externe Mitarbeitende (z. B. Freelancer) als Bereich mit weiterem Regelungs-, beziehungsweise Öffnungsbedarf betrachtet. Heute dauerten Vergabeverfahren zu lange und seien zu kompliziert. Zudem sei die Vertrauensarbeitszeit noch nicht für alle möglich, weil diese an das Lohnklassensystem gekoppelt sei. Generell müssten sich die gesetzlichen und internen Rahmenbedingungen den neuen Gegebenheiten schneller anpassen können. Denkbar wäre in diesem Kontext die Definition zeitlich befristeter Experimentierfelder, damit der Gesetzgeber für einen bestimmten Zweck einige bisherige Rahmenbedingungen außer Kraft setzen könnte und die hiermit verbundenen Effekte beurteilen und für dauerhafte Festlegungen berücksichtigen könnte (BIT (P6, P7, P9) 2018).

\subsubsection{Zukunftsperspektiven der Arbeitsmodelle im Lichte der Digitalisierung}

Nahezu alle Befragten hoben für die Zukunft eine weitere Forcierung von organisations- und unternehmensübergreifenden Zusammenarbeitsformen auf der Grundlage skillbasierter Teams und Netzwerke hervor. Von den neuen agilen Arbeitsmodellen, wie beispielsweise Holacracy oder projektbasiertes Arbeiten nach Scrum oder Kaizen werden in der Zukunft in Verbindung mit einer transparenzfördernden Digitalisierung weitere Impulse für noch mehr orts- und zeitunabhängiges sowie effizienteres Arbeiten erwartet. Die Mitarbeitenden können sich zukünftig noch mehr bei der Bearbeitung von Aufgaben und Veränderungsprozessen einbringen, mitgestalten und mitbestimmen, was zur Erhöhung der Arbeitsmotivation beitragen sollte. Ferner wird für die Zukunft ein Mehr an Optionen für den Einsatz von Freelancern erwartet (BIT (P6, P7, P9) 2018; SBB (P1, P2, P3, P5, P10) 2018; Versicherungsunternehmen (P4, P8) 2018).

Zukünftig werden die Mitarbeitenden vermutlich aufgrund weiter reduzierter Präsenzzeiten noch mehr auf Distanz geführt, und der unmittelbare persönliche Kontakt mit Teamkollegen wird geringer. Gleichzeitig steigt der Bedarf für eine engere und intensivere Zusammenarbeit, da die Aufgabenstellungen zunehmend komplexer werden und 
schneller erledigt werden müssen. Die Unternehmen werden in Zukunft vermutlich vermehrt dazu übergehen, Büroräume flexibel anzumieten, um Fixkosten zu senken. Trotzdem werden Büroarbeitsplätze am Standort voraussichtlich auch in zukünftigen Arbeitsmodellen weiterhin eine Rolle dabei spielen, wenn es um den Aufbau von informellen Beziehungen und eine Identifikation und Verbindung zum Unternehmen, respektive der Organisation geht. Bei den Befragten der SBB wurde vermutet, dass zukünftig Großunternehmen sich vermehrt in vernetzte kleinere und flexiblere Einheiten aufteilen werden. Holacracy wird von den bei der SBB interviewten Personen auch für die Zukunft vor allem in den Konzernleitungsbereichen sowie der IT und dem Human Resource Management ein hohes Effizienzverbesserungspotenzial zugeschrieben. Hiermit verbunden dürfte die Arbeit durch regelmäßige Rollenwechsel abwechslungsreicher und überflüssige Meetings und formalisierte Personalbeurteilungsformen und Personalführungsprozesse deutlich weniger werden. Allerdings ist Holacracy nicht für alle Bereiche geeignet. Die Experten der SBB rechnen für die Zukunft auch mit einem generellen Wachstum bei Mehrfachanstellungen, sodass sich das traditionelle eingleisige Arbeitgeber-Arbeitnehmerverhältnis spürbar verändern wird. Die Bedeutung von News und Feeds dürfte für die Zukunft ferner anstelle des bisherigen Mailverkehrs deutlich zunehmen (SBB (P1, P2, P3, P5, P10) 2018).

Seitens der Befragten des Versicherungsunternehmens wird ebenfalls das zu erwartende Wachstum im Freelancer-Bereich und bei den Ich-AGs betont, auch wenn es sehr wahrscheinlich nach wie vor traditionelle Beschäftigungsverhältnisse mit einer gewissen Jobsicherheit geben wird. In dem Zusammenhang kommt dann Lebensarbeitsmodellen eine wachsende Bedeutung zu (Versicherungsunternehmen (P4, P8) 2018).

Die Experten des BIT erwarten für die Zukunft unter anderem auch kostenbedingt eine wachsende und intensivere Zusammenarbeit mit Freelancern. Im öffentlich-rechtlichen Umfeld benötigt es aber hierfür klare Rahmensetzungen. Multispace-Büroräume mit Rückzugs-, Projekt- und Begegnungszonen werden vermutlich weiter ausgedehnt. Zyklische Wechsel von Teams und Projekten dürften in wachsendem Maße für das Gros der Mitarbeitenden zur Selbstverständlichkeit werden. Trotz der Entwicklungstendenz hin zu agilen Strukturen wird aber auch für die Zukunft Bedarf an coachenden, moderierenden und koordinierenden Führungspersonen gesehen (BIT (P6, P7, P9) 2018).

Potenzielle Problemfelder für die Zukunft sehen die Interviewten hinsichtlich einer reduzierten bilateralen und persönlichen Kommunikation in agileren und digitalen (Führungs-)Strukturen und damit verbundenen Unsicherheiten und Störungen im sozialen Arbeitsumfeld mancher Beschäftigter. Das Mehr an Verantwortung in instabilen Umfeldern könne bei Mitarbeitenden auch zu Überforderung oder Selbstausbeutung führen. Insofern kann das Weniger an Struktur auch zu einem Mehr an Regelungsbedarfen führen (BIT (P6, P7, P9) 2018; SBB (P1, P2, P3, P5, P10) 2018; Versicherungsunternehmen (P4, P8) 2018).

\subsubsection{Zusammenfassung der Ergebnisse}

Die befragten Großunternehmen/-organisationen setzen heute eine Bandbreite von Arbeitsmodellen mit traditionellen und agilen Elementen ein. In den Unternehmen 
kommen Jahres- und Vertrauensarbeitszeit- sowie amorphe Arbeitszeitmodelle zum Einsatz. Im Vordergrund stehen bei den Mitarbeitenden die Gleitzeit-/Jahresarbeitszeitmodelle. Vertrauensarbeitszeitmodelle sind primär für Führungskräfte mit einem definierten Gehaltsniveau relevant. In den untersuchten Organisationen wird Teilzeitarbeit, Jobsharing und mobil-flexibles Arbeiten praktiziert. Vielfach arbeiten Mitarbeitende in Projekten mit agilen Projektstrukturen. Teilweise wird mit effizienz- und flexibilitätsfördernden Methoden wie Scrum, Kaizen oder DevOps gearbeitet. Freelancing und Human-Cloud-Ansätze werden primär als Optionen für die Zukunft gesehen und sind heute noch wenig relevant. Homeoffice und Remote Working ist in der Regel in Absprache mit den Vorgesetzten möglich. Karrierebezogen kommen Einsteigerprogramme für junge Mitarbeitende und für ältere Mitarbeitende Optionen wie Bogenkarrieren und Teil- oder Frühpensionierungen zum Einsatz. Im Bereich der Personalführung kommen situativ verschiedenste Ansätze von traditionell-hierarchisch bis kooperativ zum Tragen. Der Umfang der direkten Führung wird tendenziell in Verbindung mit einem Mehr an selbstorganisierenden Teams heute schon geringer. Coachingansätze der Führung, zielorientierte holokratische Führungsperspektiven, systemische Führung und Führung auf Distanz werden, zumindest in einzelnen Bereichen, bereits gelebt. Symbolische Führung wurde in den Interviews nicht thematisiert. Alle Großunternehmen/-organisationen verfügen über Großraumbüros mit Rückzugsmöglichkeiten, Sitzungs- sowie Projekträumen. Multi-Space-Welten sind bereits Standard. Klassische Bürolösungen mit Einzelbüros werden nicht, respektive kaum mehr genutzt. Auch bei den strukturellen Lösungsansätzen für Zusammenarbeit wird situativ eine Bandbreite von traditionell-hierarchisch (bei effizienzorientierten, standardisierten Arbeiten) bis agil (bei Projektarbeiten) abgedeckt. Struktur- und Führungsmodelle hängen dabei eng miteinander zusammen. In projektorientierten Arbeitsumfeldern kommen holokratische agile Netzwerkstrukturen mit autonomen Teams zum Einsatz, die auch durch orts- und zeitunabhängiges Arbeiten gekennzeichnet sind. Auf die Einhaltung der gesetzlichen Rahmenbedingungen wird in allen Organisationen konsequent geachtet. Die technischen Voraussetzungen und die Infrastruktur für agiles Arbeiten sind in allen Organisationen vorhanden. Wenn möglich, werden Sonderbewilligungen für Arbeitszeitflexibilisierungen eingeholt. Die realisierten Arbeitsmodelle scheinen insgesamt in ihrem gegenwärtigen Mix aus stabilitäts- und flexibilitätsbezogenen Komponenten gut zu funktionieren und werden von den Mitarbeitenden großteils als passend wahrgenommen.

Die derzeit umgesetzten Arbeitsmodelle haben situationsabhängige Vor- und Nachteile. Chancen für die Zukunft werden vor allem in Verbindung mit agilen holokratischen Arbeitsmodellen gesehen, die flexibler und kundenorientierter sind und sich voraussichtlich weiter ausdehnen werden. Die neueren agilen Ansätze der Zusammenarbeit sind nicht nur für die Wettbewerbsfähigkeit des Unternehmens relevant, sondern für neue jüngere Mitarbeitende auch eine wichtige Komponente der Arbeitgeberattraktivität. Ein intensiverer Einsatz von digitalen Technologien ist auch mit einer flexibleren Ausgestaltbarkeit der Arbeitsmodelle verbunden, schafft Freiräume für Führungsarbeit, reduziert die Raumkosten, erhöht die Vereinbarkeit von Freizeit und Beruf, verbessert 
die Datenverfügbarkeit, die Motivation und senkt die Wegzeiten der Mitarbeitenden. Mit der Einführung mobilerer und flexiblerer agiler Strukturen einher geht allerdings auch das Risiko einer Überforderung von Mitarbeitenden und einer Vermischung von Arbeitsund Freizeitsphären, die sich auch negativ auf Commitment, Motivation und Gesundheit der Mitarbeitenden auswirken kann. In dem Zusammenhang ist auch mit auf Ängsten und Gewohnheit basierendem Widerstand von Mitarbeitenden bei der Einführung agiler Arbeitsmodelle zu rechnen.

Für die Anpassung bestehender und Einführung neuer flexiblerer Arbeitsmodelle im Kontext der Veränderung der digitalen Rahmenbedingungen gibt es derzeit keine Standardrezepte. Dies erfolgt sehr kontextabhängig, eher stufen- und bereichsweise, oftmals in Verbindung mit geänderten Anforderungen der Kunden und Mitarbeitenden. Die Einführung neuer Arbeitsmodelle erfolgt dabei tendenziell eher top-down, wird aber in der Regel mit Bottom-up-Kopplungen kombiniert. Externe Beratungen spielen bei der Einführung in den befragten Organisationen eher keine Rolle. Bei der üblicherweise als Projekt erfolgenden stufenweisen Einführung neuer Formen der Zusammenarbeit sind die betroffenen Mitarbeitenden mittels Bedürfniserhebung, Überzeugungsarbeit und Mitwirkungsoptionen einzubinden. Die neuen Arbeitsmodelle müssen zur Arbeitstätigkeit und Kultur passen, was in Pilotumsetzungen getestet werden kann. Die Führungskräfte sollten die eingeforderten neuen Arbeitsweisen als Vorbilder selbst auch vorleben. Darüber hinaus ist für den Erfolg das Erkennen eines klaren kunden-, mitarbeitenden- und effizienzbezogenen Mehrwerts von Bedeutung. Es sollte insofern für alle Betroffenen deutlich werden, dass es bei der Einführung eines neuen Arbeitsmodells nicht um das bloße Aufgreifen eines Managementtrends geht, sondern um eine Verbesserung der Wettbewerbsfähigkeit. Pointiert können somit unter Bezugnahme auf die erfolgten Interviews folgende Handlungsempfehlungen für die Einführung und Änderung neuer Arbeitsmodelle abgeleitet werden:

- Nachvollziehbare Begründungen für den Wandel (Sinnstiftung).

- Kontextabhängige, iterative Projektimplementierung (situativer Projektansatz).

- Stimmige Veränderung des Mindsets (Struktur-Kultur-Fit).

- Einbindung und Mitgestaltung der Mitarbeitenden (Faktor Mensch).

Die Digitalisierung ermöglicht und beschleunigt die Einführung von agilen Projekt- und Netzwerkstrukturen und systemischer Führung. Sie fördert orts- und zeitunabhängiges, organisationsübergreifendes und interdisziplinäres Zusammenarbeiten etwa über Cloudund mobile Kommunikationslösungen wie Feeds und News. Sie ermöglicht auch neue Formen der Zusammenarbeit im Arbeitssegment der selbstständig mitarbeitenden Freelancer und bei Mehrfachbeschäftigten. Neben der Flexibilisierung der Arbeit geht mit der Digitalisierung auch eine potenzielle Flexibilisierung der Strukturen einher, die für Großunternehmen zum Beispiel in die Aufteilung in kleinere Netzwerkeinheiten münden könnte. So kann den gestiegenen Anforderungen der Kunden und Mitarbeitenden 
besser entsprochen werden. Digitalisierung begünstigt ferner den Einsatz moderner, auf Flexibilität abhebender Arbeitsmethoden wie Scrum oder Design Thinking.

Die neuen Arbeitsmodelle sind mit zusätzlichen Freiräumen verbunden, für die ein stabiler Handlungsrahmen von Bedeutung ist. Die heutigen gesetzlichen Rahmenbedingungen in der Schweiz werden teilweise nur bedingt den Anforderungen von agileren und flexibleren Arbeitsmodellen der Zukunft gerecht. Als relevante Regelungsfelder mit Entwicklungspotenzial erscheinen etwa die zunehmende Verschmelzung von beruflichen und privaten Lebenssphären, die derzeit restriktiven Vorgaben hinsichtlich Wochenend- und Sonntagsarbeit, die Rahmenbedingungen für eine Zusammenarbeit mit Freelancern sowie für Mehrbeschäftigungsverhältnisse, Jobsharing oder lebensphasenorientierte Rahmensetzungen.

\subsubsection{Schlussbetrachtung und Ausblick}

Die empirische Untersuchung anhand der drei interviewbasierten Fallstudien bestätigt im Wesentlichen das Bild hinsichtlich der Relevanz der Digitalisierung für neue Arbeitsmodelle und deren Umsetzungsformen sowie Entwicklungstendenzen in Richtung Agilität, Flexibilisierung und Mobilisierung der Arbeit. Diese Tendenzen entsprechen großteils auch den veränderten heutigen Bedürfnislagen der Mitarbeitenden und stehen in einem engen Zusammenhang mit einer effizienteren und fokussierteren Arbeitsdurchführung. Mit den neuen Arbeitsformen einher geht auch eine Flexibilität in Bezug auf sich im Phasenverlauf des Erwerbslebens verändernde Bedürfnislagen der Mitarbeitenden. Die untersuchten Organisationen haben in einigen Anwendungsbereichen und -dimensionen bereits einen hohen Entwicklungsstand erreicht und entwickeln ihre Arbeitsmodelle kontinuierlich weiter. „Neue Arbeitsmodelle“ sind insofern heute bereits Realität. Die inhaltlichen Aussagen der befragten drei Fallstudienpartner weisen eine große Schnittmenge für nahezu alle angeschnittenen Themenfelder auf. Angesichts der hohen Kontingenzabhängigkeit der Thematik, der Fallzahl und der begrenzten Anzahl der Interviewpartner ist dennoch eine Generalisierung der Erkenntnisse nur bedingt möglich. Insofern ist die Untersuchung als ein methodisch fundierter deskriptiver Pinselstrich von vielen zu verstehen, die skizzenhaft versuchen, ein mehrdimensionales Bild von der Arbeitswelt von morgen zu zeichnen.

Auch wenn heute noch niemand genau vorhersagen kann, wie Arbeitsmodelle in Verbindung mit der voranschreitenden Digitalisierung in zehn oder fünfzehn Jahre voraussichtlich aussehen werden, so scheinen die angeführten Entwicklungstendenzen und die hiermit verbundenen Schlagworte „Kundenzentrierung“, „Agilität“, „Selbstorganisation“, „Flexibilisierung“, „Dynamisierung“, „Mobilität“, „Teamorientierung“, „Kollaboration“ und „Vernetzung“ der Arbeit doch zumindest einen möglichen Entwicklungskorridor aufzuzeigen. Heute noch bestehende Grenzziehungen zwischen Arbeits- und Privatwelt sowie Unternehmensinnen- und -außenwelten scheinen zunehmend fluid $\mathrm{zu}$ werden, 
es wird aber voraussichtlich dennoch kontextabhängig auch auf absehbare Zeit noch Arbeitsmodelle mit effizienzorientierten und innovationsfördernden Dimensionsausprägungen in standortbasierten Arbeitswelten geben.

\section{Literatur}

Arbeitgeberverband Basel. (2014). Personalradar. http://www.personalradar.ch/arbeitsrecht-kategorien/arbeitszeitmodelle-und-ihre-rechtlichen-folgen/10052. Zugegriffen: 28. März 2018.

Auf der Maur, M. (2017). Büroflächenmarkt Schweiz: Coworking Spaces liegen im Trend. Schweizer Immobilienbrief, 20, 7-9.

Berghaus, S., Back, P., \& Kaltenrieder, B. (2017). Digital Maturity \& Transformation Report 2017. St. Gallen: Institut für Wirtschaftsinformatik, Universität St. Gallen, Crosswalk AG.

Berlin, B. B. (2016). Mittelstand im Wandel - Wie ein Unternehmen seinen digitalen Reifegrad ermitteln kann. Berlin: HBP University Press.

Blaeser-Benfer, D., \& Pollety, W. (2017). Digitalisierung, Betriebliche Handlungsfelder der Unternehmensentwicklung. Frankfurt a. M.: Frankfurter Societäts-Medien GmbH.

Bürgi und Nägeli Rechtsanwälte. (o. J.). Arbeitsrecht - Job sharing. https://www.arbeits-recht.ch/ job-sharing. Zugegriffen: 3. Apr. 2018.

Deflorin, P., Hauser, C., \& Scherrer-Rathje, M. (2015). Schweizer Unternehmen sehen Digitalisierung als Chance. o. $\mathrm{O}$.

Deloitte. (2016). Deloitte. https://www2.deloitte.com/content/dam/Deloitte/ch/Documents/consumer-business/ch-de-cip-swiss-transformation.pdf. Zugegriffen: 27. März 2018.

Digital Transformer Institute. (2017). Digitaler Reifegrad Check. http://www.digitaltransformerinstitute.ch/services/reifegrad-check/\#1452765442453-b921a1fb-6ec8. Zugegriffen: 14. Febr. 2018.

Disler, A. (2018). adigiconsult GmbH. https://www.adigiconsult.ch/6-treiber-der-digitalisierung/. Zugegriffen: 12. Apr. 2018.

Frey, C., \& Osborne, M. A. (2013). The future of employment. How suspectible are jobs to computerisation? Oxford Martin School (OMS), Working Paper. Oxford: University of Oxford.

Genner, S., Probst, L., Huber, R., Werkmann-Karcher, B., Grundrum E., Majkovic, A.-L. (2017). IAP Studie 2017. Der Mensch in der Arbeitswelt 4.0. Zürich: IAP Institut für Angewandte Psychologie der ZHAW Zürcher Hochschule für Angewandte Wissenschaften.

Glaser, N. (2016). Markenkonstrukt. https://www.markenrebell.de/2016/07/19/so-wirkt-sich-die-digitalisierung-aus/\#.Wvhfh2cUmM8. Zugegriffen: 2. Apr. 2018.

Glogner-Pilz, P. (2012). Publikumsforschung - Grundlagen und Methoden. Wiesbaden: Springer Gabler.

Grannemann, U. (2015). Leadion. https://www.leadion.de/2015/10/18/kooperativer-bis-autoritaerer-fuehrungsstil-das-kontinuummodell-von-tannenbaum-und-schmidt/. Zugegriffen: 6. Apr. 2018.

Institut für Wirtschaftsinformatik. (2017). Digital Maturity \& Transformation Studie. https://aback. iwi.unisg.ch/kompetenz/digital-maturity-transformation/. Zugegriffen: 12. Febr. 2018.

Ittermann, P., \& Niehaus, J. (2015). Digitalisierung industrieller Arbeit. Baden-Baden: Nomos.

Leyh, C., Schäffer, T., \& Forstenhäusler, S. (2016). SIMMI 4.0 - Vorschlag eines Reifegradmodells zur Klassifikation der unternehmensweiten Anwendungssystemlandschaft mit Fokus Industrie 4.0. Dresden: o. V.

Lienhart, A. (2015). WEKA. https://www.weka.ch/themen/personal/arbeitszeit-und-absenzen/ arbeitszeitmodelle/article/vertrauensarbeitszeit-in-der-schweiz-weshalb-das-vertrauen-genaue-planung-und-kontrolle-braucht/. Zugegriffen: 28. März 2018. 
Meyer, M. (2016). Bilanz. https://www.bilanz.ch/management/das-ende-der-hierarchien-holacracyschafft-den-chef-ab-761343. Zugegriffen: 07. Apr. 2018.

Moneyhouse. (2017). 99,8 Prozent der Unternehmen in der Schweiz sind KMU. http://ratgeber. moneyhouse.ch/article/998-prozent-der-unternehmen-in-der-schweiz-sind-kmu/. Zugegriffen: 25. März 2018.

Mühlenbeck, F. (2017). Die Wirkungsweise der digital Transformation - Ein Modell. http://transformieren.com/digital-transformation-wirkungsweise-modell/. Zugegriffen: 25. März 2018.

Neufeind, M. (2016). Generation Y, Wie eine kreative Generation heute Grenzen verschiebt. Berlin: De Gruyter.

Raveling, J. (2017b). Wirtschaftsförderung Bremen GmbH. https://www.wfb-bremen.de/de/page/ stories/digitalisierung-industrie40/geschichte-der-digitalisierung-teil-zwei. Zugegriffen: 11. Febr. 2018.

Reynard, \& Derder. (2017). Kurzfassung: Auswirkungen der Digitalisierung auf Beschäftigung und Arbeitsbedingungen - Chancen und Risiken. o. O.: o. V.

Röbken, P. D., \& Wetzel, K. (2016). Universität Oldenburg. https://www.uni-oldenburg.de/filead$\mathrm{min} /$ user_upload/c31/Studiengaenge/BABusinessAdmin/Download/Leseproben/bba_leseprobe_ quli_quanti_forschungsmethoden.pdf. Zugegriffen: 12. Apr. 2018.

Rotter, D. (2017). Beraternews.net. http://www.beraternews.net/wirtschaft/unternehmen-nutzenchancen-der-digitalisierung-kaum-35712279/. Zugegriffen: 27. März 2018.

Ruoss, S. (2015). Digitale transformation. https://www.firegroup.ch/de/blog/digitale-transformation-teil-8. Zugegriffen: 14. Febr. 2018.

Sattelberger, T., Welpe, P., \& Boes, P. (2015). Das demokratische Unternehmen. Freiburg: Haufe-Lexware.

Schallmo, D., \& Rusnjak, A. (2017). Roadmap zur Digitalen Transformation von Geschäftsmodellen. In D. Schallmo, A. Rusnjak, J. Anzengruber, T. Werani, \& M. Jünger (Hrsg.), Digitale Transformation von Geschäftsmodellen - Grundlagen, Instrumente und Best Practices. Wiesbaden: Springer Gabler.

Schwab, K. (2016). Die Vierte Industrielle Revolution. München: Pantheon.

Schweizer Verband der Telekommunikation. (2015). asult-Bulletin. Bern: KMU und Digitalisierung.

Schweizerische Bundesbahnen (SBB AG), \& Swisscom (Schweiz) AG. (2013). Workanywhere. Bern: PrintMedia Services.

Schweizerische Eidgenossenschaft. (2015a). Mehrfachbeschäftigung. https://www.kmu.admin. $\mathrm{ch} / \mathrm{kmu} / \mathrm{de} / \mathrm{home} /$ praktisches-wissen/personal/arbeitsrecht/mehrfachbeschaeftigung.html. Zugegriffen: 3. Apr. 2018.

Schweizerische Eidgenossenschaft. (2015b). Nacht- und Sonntagsarbeit. https://www.kmu.admin. $\mathrm{ch} / \mathrm{kmu} / \mathrm{de} / \mathrm{home} /$ praktisches-wissen/personal/arbeitsrecht/nacht-und-sonntagsarbeit.html. Zugegriffen: 3. Apr. 2018.

Schweizerische Eidgenossenschaft. (2016). Arbeitszeiterfassung: Pflicht für Arbeitgebende. https://www.kmu.admin.ch/kmu/de/home/praktisches-wissen/personal/arbeitsrecht/arbeitszeiterfassung-pflicht-arbeitgeber.html. Zugegriffen: 3. Apr. 2018.

Schweizerische Eidgenossenschaft. (2018a). Digitale Wirtschaft. https://www.seco.admin.ch/ seco/de/home/wirtschaftslage—wirtschaftspolitik/wirschaftspolitik/digitalisierung.html. Zugegriffen: 8. Apr. 2018.

Schweizerische Eidgenossenschaft. (o. J.a.). Arbeitsrecht: die gesetzlichen Grundlagen. https:// www.kmu.admin.ch/kmu/de/home/praktisches-wissen/personal/arbeitsrecht.html. Zugegriffen: 3. Apr. 2018.

Schweizerische Eidgenossenschaft. (o. J.c.). Überstunden. https://www.kmu.admin.ch/kmu/de/ home/praktisches-wissen/personal/arbeitsrecht/ueberstunden.html. Zugegriffen: 3. Apr. 2018. 
Süddeutsche Zeitung. (o. J.). Digitales Morgen. http://www.sueddeutsche.de/thema/Digitales_Morgen. Zugegriffen: 2. Apr. 2018.

Wissen. Weiterbildung. Lösungen (WEKA). (2016). Führungsstil: Übersicht und Anwendungstipps. https://www.weka.ch/themen/fuehrung-kompetenzen/mitarbeiterfuehrung/fuehrungsinstrumente/article/fuehrungsstil-uebersicht-und-anwendungstipps/. Zugegriffen: 6. Apr. 2018.

Witzig the office company. (2014). FlexWork - die Vorteile des flexiblen Arbeitens. https://www. witzig.ch/de/post/20140406/flexwork-die-vorteile-des-flexiblen-arbeitens. Zugegriffen: 7. Apr. 2018.

Work Smart Initiative. (2018). Work smart initiative. http://work-smart-initiative.ch/de/\%C3\%BCberuns/charta-unterzeichnen/. Zugegriffen: 27. Febr. 2018.

Zinser, S., \& Boch, D. (Hrsg.). (2007). Flexible Arbeitswelten. Zürich: vdf Hochschulverlag AG an der ETH Zürich.

Zobrist, L., \& Grampp, D. (2016). Der Arbeitsplatz der Zukunft, Wie digitale Technologie und Sharing Economy die Schweizer Arbeitswelt verändern. o. O.: Deloitte AG.

\section{Interviews}

Bundesamt für Informatik und Telekommunikation BIT (P6). (2018). Digitalisierung und Arbeitsmodelle, Interview vom 06.04.2018.

Bundesamt für Informatik und Telekommunikation BIT (P6, P7, P9). (2018). Digitalisierung und Arbeitsmodelle, Interviews vom April bis Mai 2018.

Schweizerische Bundesbahnen (SBB AG) (P1). (2018). Digitalisierung und Arbeitsmodelle, Interview vom 23.03.2018.

Schweizerische Bundesbahnen (SBB AG) (P1, P2, P3, P5, P10). 2018. Digitalisierung und Arbeitsmodelle, Interviews von März bis April 2018.

Schweizerische Bundesbahnen (SBB AG) (P2). (2018). Digitalisierung und Arbeitsmodelle, Interview vom 26.03.2018.

Versicherungsunternehmen (P4). (2018). Digitalisierung und Arbeitsmodelle, Interview vom 01.05.2018.

Versicherungsunternehmen (P4, P8). (2018). Digitalisierung und Arbeitsmodelle, Interviews im Mai 2018.

Schellinger, Jochen (Prof. Dr./jochen.schellinger@bfh.ch) Studiengangsleiter Master of Science in Business Administration an der Berner Fachhochschule. Lehr- und Forschungstätigkeiten in den Bereichen Strategisches Management, Marketing und Personalmanagement. Davor 15 Jahre Praxistätigkeit bei der Deutschen Sparkassenorganisation, im Haniel-Konzern, an der Universität Tübingen sowie in der Daimler AG.Huynh, Gia Le (M.Sc. BA/gia-le.huynh@gmx.net) Masterstudium in Betriebsökonomie mit Vertiefung in Corporate/Business Development an der Berner Fachhochschule. Langjährige Tätigkeit in Anlagensteuerung, LifeCycle- und Produktmanagement, Leistungscontrolling sowie Strategie- und Organisationsprojekten. Aktuell bei der Schweizerischen Bundesbahnen AG als Senior strategische Anlagenmanagerin im Kundenanlagen- und FacilityManagement. 
Open Access Dieses Kapitel wird unter der Creative Commons Namensnennung 4.0 International Lizenz (http://creativecommons.org/licenses/by/4.0/deed.de) veröffentlicht, welche die Nutzung, Vervielfältigung, Bearbeitung, Verbreitung und Wiedergabe in jeglichem Medium und Format erlaubt, sofern Sie den/die ursprünglichen Autor(en) und die Quelle ordnungsgemäß nennen, einen Link zur Creative Commons Lizenz beifügen und angeben, ob Änderungen vorgenommen wurden.

Die in diesem Kapitel enthaltenen Bilder und sonstiges Drittmaterial unterliegen ebenfalls der genannten Creative Commons Lizenz, sofern sich aus der Abbildungslegende nichts anderes ergibt. Sofern das betreffende Material nicht unter der genannten Creative Commons Lizenz steht und die betreffende Handlung nicht nach gesetzlichen Vorschriften erlaubt ist, ist für die oben aufgeführten Weiterverwendungen des Materials die Einwilligung des jeweiligen Rechteinhabers einzuholen.

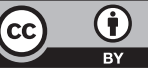

